\title{
OPTIMAL CONTROL OF A SIMPLIFIED NATURAL CONVECTION-RADIATION MODEL*
}

\author{
RENÉ PINNAU ${ }^{\dagger}$ AND OLIVER TSE
}

\begin{abstract}
The study of optimal control problems for the two fields of fluid flow and radiation gained considerable attention during the last decade. In this paper we present a comprehensive analysis of an optimal boundary control for a combined natural convection-radiation model, which has applications in the design of combustion chambers or for the control of cooling processes, glass production, or crystal growth. The model under investigation consists of the transient Boussinesq system coupled with a nonlinear heat equation and the $\mathrm{SP}_{3}$ model for radiation. First, we show the existence of unique, regular solutions for this forward system, which is challenging due to the cross-diffusion character of the $\mathrm{SP}_{3}$ model, since it does not allow for the application of standard maximum principles. Second, we show the existence of an optimal boundary control and analyze the linearized state system, which yields the existence of adjoint states and the differentiability of the control-to-state map.
\end{abstract}

Key words. Natural convection, radiation, $\mathrm{SP}_{N}$-approximation, optimal boundary control, KKT system, adjoints, analysis.

AMS subject classifications. 35K55, 49K20, 80A20.

\section{Introduction}

At very high temperatures, like in glass production or combustion chambers for gas turbines, radiation plays a dominant role in the energy balance (cf. [12]). In contrast to diffusive heat conduction, this yields a more complex model, which can only be simulated with standard software in limit cases, as pure surface radiation or radiation in optically thick media. In semitransparent media (cf. [36, Chapter 12]) such as glass melts or gas, however, one cannot disregard volume radiation of heat from the interior. Therefore one would require sophisticated models, which involve radiating and conducting fluids. One adequate model for the description of these media is a system of equations consisting of the radiative heat transfer (RHT) system and the hydrodynamic equations (cf. [36, Chapter 9]). Due to the high numerical complexity of simulations which include radiative effects, there is presently a whole hierarchy of approximate models available, which allows one to reduce the numerical costs significantly and still reproduce the main physical phenomena.

Here, we will focus on the diffusive-type $\mathrm{SP}_{3}$-system and show the applicability of this system in the context of coupled radiation, convection, and diffusion problems. So far, the optimal control of conducting, convecting, radiating fluids has not been extensively studied in the literature. Nevertheless, there are publications on the respective sub-problems, namely on the optimal control of RHT and on the hydrodynamic equations independently.

A first paper which focused on the identification of the boundary function for the RHT equation is [3]. There, the optimality system was analyzed via the adjoint approach. A first numerical treatment of this problem based on a Picard iteration for the first-order optimality system can be found in [4]. Similar problems were considered

\footnotetext{
${ }^{*}$ Received: February 9, 2012; accepted (in revised form):July 23, 2012. Communicated by Shi Jin.

${ }^{\dagger}$ Fachbereich Mathematik, Technische Universität Kaiserslautern, Erwin Schrödinger Straße Kaiserslautern, 67663, Germany (pinnau@mathematik.uni-kl.de).

${ }^{\ddagger}$ Fachbereich Mathematik, Technische Universität Kaiserslautern, Erwin Schrödinger Straße Kaiserslautern, 67663, Germany (tse@mathematik.uni-kl.de).
} 
for stationary nonlocal surface radiation in $[32,33,34]$ or for the optimal control of glass melts in [13]. The publications [39, 41, 46] focus on the optimal boundary control of the $\mathrm{SP}_{1}$-system to influence the cooling temperature during the process of glass cooling to avoid thermal stresses. This yields minimization problems restricted by systems of PDEs. To deal with the problem, descent algorithms were used, where the derivative information was calculated via the adjoint equations (see also [49]). A comprehensive analysis for the optimal boundary control of the $\mathrm{SP}_{1}$-system can be found in [38], where the forward problem is analyzed and the existence of minimizers as well as the existence and uniqueness of adjoint states is proven.

Contributions to the development of analytical methods and numerical algorithms for optimal control problems for the Navier-Stokes system can be found, e.g., in $[1,20,21,22,23,24]$, where the authors emphasized the development of mathematical methods in flow control and discrete concepts for PDE constrained optimization. Optimal boundary control for the Boussinesq-approximation can be found in $[8,9]$, where Bolderini et al. also considered temperature dependent coefficients. Boundary temperature control for the time dependent Boussinesq-approximation equations was numerically investigated in [7]. On account of progress previously mentioned, one can go a step further and bring together the fields of optimal control of RHT and optimal control of the hydrodynamic equations, which may, e.g., be applied to the optimal stirring of glass melts in a melting furnace.

We begin by stating the simplified natural convection-radiation model and describing the optimal control problem that we wish to solve. Section 2 is dedicated to the analysis of the state system, i.e., we show the unique solvability of the simplified natural convection-radiation model and derive a priori estimates. In Section 3, we prove the existence of an optimal control of the generic minimization problem (1.2). Finally, we show the unique solvability of the linearized system along with its adjoint, and derive regularity results for the control-to-state map, which is essential for the characterization of local minimizers.

1.1. Mathematical problem description. The Boussinesq-system of hydrodynamics equations (cf. [10,37]) arises from a zero order approximation of the coupling between the Navier-Stokes equations and the thermodynamic equation (cf. [35]). The Boussinesq-approximations can best be summarized by two statements: (1) The fluctuations in density, which appear with the advent of motion, result principally from thermal (as apposed to pressure) effects. (2) In the equations for the rate of change of momentum and mass, density variations may be neglected except when they are coupled to the gravitational acceleration in the buoyancy force.

We study the Boussinesq-system for a conducting fluid (cf. [10, 35, 37]) where the temperature is influenced by radiation modeled via the diffusive $\mathrm{SP}_{3}$-approximation (cf. [40]), which we call the simplified natural convection-radiation (SNCR) system. The domain $\Omega \subset \mathbb{R}^{2}$ consist of a gray, absorbing, emitting, and scattering fluid surrounded by rigid walls $\Gamma$. The fluid is considered to be Newtonian and all the thermophysical properties are assumed to be constant, except for the density in the buoyancy term. Compression effects and viscous dissipation are also neglected.

The scaled SNCR system consists of the Navier-Stokes equations

$$
\begin{aligned}
\nabla \cdot v & =0, & & \\
\partial_{t} v+v \cdot \nabla v-\operatorname{Pr} \nabla \cdot \nu \nabla v+\nabla p & =\operatorname{Ra} \operatorname{Pr} \vartheta e_{2}, & & \text { in } Q:=(0, T) \times \Omega, \\
v & =0, & & \text { on } \Sigma:=(0, T) \times \Gamma,
\end{aligned}
$$


coupled with the thermodynamic equation

$$
\begin{aligned}
\partial_{t} \vartheta+v \cdot \nabla \vartheta-\nabla \cdot \kappa \nabla \vartheta+\mathrm{Pl}^{-1} \nabla \cdot q & =0, & & \text { in } Q, \\
n \cdot \kappa \nabla \vartheta+\gamma(\vartheta-u) & =0, & & \text { on } \Sigma,
\end{aligned}
$$

where $e_{2}$ is a unit vector. The state vectors are the velocity vector $v$, the temperature $\vartheta$, and the pressure $p$. The nonnegative parameters are the Prandtl number Pr, the Planck number $\mathrm{Pl}$, and the Rayleigh number $\mathrm{Ra}$, as well as the scaled kinematic viscosity $\nu$, scaled thermal diffusivity $\kappa$, and boundary coefficient $\gamma$. We assume throughout that $\nu, \kappa \in L^{\infty}(\Omega)$ are bounded from below by positive constants $\underline{\nu}, \underline{\kappa}>0$ respectively, and $\gamma \in L^{\infty}(Q)$ with $\gamma \geq 0$ but $\gamma \neq \equiv 0$, where $\gamma=0$ on a subset of the boundary corresponds to the homogeneous Neumann boundary condition and $\gamma>0$ corresponds to the Robin-type boundary condition.

The dimensionless radiative heat flux is given by

$$
\nabla \cdot q=\sigma_{a}\left(I_{b}(\vartheta)-\phi\right),
$$

where $\phi$ is the total incident radiation, which is computed via the $\mathrm{SP}_{3}$-approximation

$$
\begin{aligned}
-\nabla \cdot \frac{\epsilon^{2}}{3 \sigma_{t}}(\nabla \phi+2 \nabla \xi)+\sigma_{a}\left(\phi-I_{b}(\vartheta)\right) & =0, \\
-\nabla \cdot \frac{\epsilon^{2}}{3 \sigma_{t}}\left(\frac{2}{5} \nabla \phi+\frac{11}{7} \nabla \xi\right)+\sigma_{t} \xi & =0,
\end{aligned} \quad \text { in } Q,
$$

with boundary conditions

$$
\begin{aligned}
n \cdot \frac{\epsilon^{2}}{3 \sigma_{t}}(\nabla \phi+2 \nabla \xi)+\alpha_{1,1}\left(\phi-I_{b}(u)\right)+\alpha_{1,2} \xi & =0, \\
n \cdot \frac{\epsilon^{2}}{3 \sigma_{t}}\left(\frac{2}{5} \nabla \phi+\frac{11}{7} \nabla \xi\right)+\alpha_{2,1}\left(\phi-I_{b}(u)\right)+\alpha_{2,2} \xi & =0,
\end{aligned}
$$

with the scaled Planck function given by $I_{b}(w)=(w+1)^{4}$, extinction and absorption coefficients $\sigma_{t}, \sigma_{a} \in L^{\infty}(Q)$, which are bounded from below by positive constants $\underline{\sigma}_{t}, \underline{\sigma}_{a}>0$ respectively, and boundary parameters $\alpha_{i, j}$ depending on the reflectivity $\rho$ of the material on the boundary $\Gamma$. We refer the reader to [14] for the formulation of these dimensionless equations. Here, we can already see the problems that may arise due to the presence of cross-diffusion and boundary coupling in the coupled equations (1.1e, 1.1f), which make the analysis for these equations non-trivial. We will point out these problems in the next section.

To formulate a well-posed problem, system (1.1) must be solved within a bounded time interval $I:=(0, T) \subset \mathbb{R}$ with $T<\infty$ and a bounded domain $\Omega \subset \mathbb{R}^{2}$ with sufficiently smooth boundary $\Gamma$, which we assume to be at least Lipschitz-continuous, while subjected to given initial conditions. The restriction to domains in $\mathbb{R}^{2}$ is mainly due to the lack of analytical results available for the Navier-Stokes equations in $\mathbb{R}^{3}$.

1.2. Optimal control problem. We denote the solutions of the state equation (1.1) by state vectors $y=(v, \vartheta, \Phi)$, where $v$ is the velocity vector, $\vartheta$ the temperature and $\Phi=(\phi, \xi)$ are the solutions of the $\mathrm{SP}_{3}$-approximation. The task at hand is to find a boundary control $u$ representing the ambient temperature by minimizing a given cost functional $J$ with the hope of either tracking a desired state $y_{\mathrm{d}}$ such as the 
temperature profile $\vartheta_{\mathrm{d}}$ or optimally stirring glass melts in a melting furnace, which may be described by the following minimization problem:

$$
\begin{aligned}
& \min J(u, y)=J_{1}(y)+J_{2}(v(T), \vartheta(T))+J_{3}(u) \\
& \text { with respect to }(u, y) \quad \text { such that } \quad(1.1) .
\end{aligned}
$$

We consider two simple examples for the cost functional $J$ that are applicable to the glass manufacturing industry.

1.2.1. Problem 1: State tracking. The first example we want to consider involves tracking a desired temperature distribution $\vartheta_{\mathrm{d}}$ of the system, i.e. given an initial state of the system $y_{0}$ we would like to steer the system into a state $y_{*}$ having the temperature distribution $\vartheta_{*} \approx \vartheta_{\mathrm{d}}$. There are several ways to achieve this, of which two are stated here. More specifically, we want to minimize the cost functional

$$
J(u, \vartheta)=\underbrace{\frac{\delta_{1}}{2}\left\|\vartheta-\vartheta_{\mathrm{d}}\right\|_{2, Q}^{2}}_{J_{1}(y)}+\underbrace{\frac{\delta_{2}}{2}\left\|\vartheta(T)-\vartheta_{\mathrm{d}}\right\|_{2, \Omega}^{2}}_{J_{2}(\vartheta(T))}+\underbrace{\frac{\lambda}{2}\|u\|_{W}^{2}}_{J_{3}(u)},
$$

where the first two terms $J_{1}$ and $J_{2}$ describe the error between the simulated temperature distribution $\vartheta$ and desired temperature distribution $\vartheta_{\mathrm{d}}$, and the third term $J_{3}$ penalizes the cost of the control $u$, with $\lambda$ as a regularization parameter. Here, $W$ is a suitable space of controls. For the case where $\delta_{2}=0$ we require the functional to be minimal throughout the entire time horizon, whereas for the case $\delta_{1}=0$ we only require that the desired state be achieved at the given time $T$. A linear combination of both cases may also achieve desirable results.

1.2.2. Problem 2: Maximizing vorticity. In this example we would like to have a good mixture of the glass melt in the cavity. This may be expressed mathematically as requiring the vorticity of the fluid to be at its maximum, which leads to the problem of minimizing the cost functional

$$
J(u, v)=\underbrace{-\frac{\delta}{2}\|\nabla \times v\|_{2, Q}^{2}}_{J_{1}(y)}+\underbrace{\frac{\lambda}{2}\|u\|_{W}^{2}}_{J_{3}(u)},
$$

where the first term $J_{1}$ rewards the vorticity of the fluid and the second term $J_{3}$ penalizes the cost of the control $u$, with $\lambda$ as a regularization parameter. Similarly, $W$ is a suitable space of controls.

1.3. Notation. Let $G \in\{\Omega, \Gamma, Q, \Sigma\}, k \in \mathbb{N}$, and $p \in[1, \infty]$. We denote the Lebesgue spaces by $\left(L^{p}(G),\|\cdot\|_{p, G}\right)$ and the Sobolev spaces by $\left(W^{k, p}(G),\|\cdot\|_{W^{k, p}(G)}\right)$ (c.f. [2]). The duality pairing of a Banach space $X$ with its dual $X^{*}$ is given by $\langle\cdot, \cdot\rangle_{X}$; if the spaces involved are clear, we simply write $\langle\cdot, \cdot\rangle$. For a Hilbert space $H(G)$, its inner product is denoted by $(\cdot, \cdot)_{G}$. We denote by $p^{\prime}$ the conjugate for $p$, i.e., $(p-1) p^{\prime}=p$ such that $L^{p}(G)^{*} \cong L^{p^{\prime}}(G)$. For the special case $p=2$ we use $H^{k}(G)$ to denote $W^{k, 2}(G)$. Further, let $H_{0}^{k}(G)$ be the closure of $\mathcal{C}_{0}^{\infty}(G)$ with respect to the $H^{k}(G)$-norm. Its dual space is denoted by $H^{-k}(G)$. For a bounded interval $I$ and Banach space $X$, we denote the Lebesgue-Bochner space by $L^{p}(I ; X)$ and the SobolevBochner space by $W^{k, p}(I ; X)$ (c.f. [51]). In the case $X=L^{q}(G)$, we denote the norm of $L^{p}\left(I ; L^{q}(G)\right)$ by $\|\cdot\|_{q, p, G}$. Recall that $L^{p}\left(I ; L^{p}(\Omega)\right) \cong L^{p}(Q)$. Furthermore, we denote by $\mathcal{C}_{0}^{\infty}(G, \operatorname{div})=\left\{w \in \mathcal{C}_{0}^{\infty}(G) \mid \nabla \cdot w=0\right\}$ the space of infinitely differentiable divergence 
free vector fields with compact support, and by $\mathcal{H}, \mathcal{H}^{1}$ the closure of $\mathcal{C}_{0}^{\infty}(G$, div) in $\left[L^{2}(G)\right]^{2}$ and $\left[H_{0}^{1}(G)\right]^{2}$ with their respective norms (c.f. [44]), and the dual of $\mathcal{H}^{1}$ is denoted by $\mathcal{H}^{-1}$. For notational convenience we denote

$$
\begin{array}{cc}
V=L^{2}\left(I ; H^{1}(\Omega)\right), & \mathcal{W}(V)=V \cap H^{1}\left(I ; H^{-1}(\Omega)\right) \\
\mathcal{V}=L^{2}\left(I ; \mathcal{H}^{1}\right), & \mathcal{W}(\mathcal{V})=\mathcal{V} \cap H^{1}\left(I ; \mathcal{H}^{-1}\right)
\end{array}
$$

Generic positive constants are denoted by $c_{i}$ and may depend on the domain $\Omega$ or boundary $\Gamma$ considered. For vectors the lower index refers to the respective component. The upper parenthesized index denotes an iteration step. Overbar and underbar of functions refer to the upper and lower bounds of essentially bounded functions respectively when either exists.

\section{The state system}

The mathematical investigation of the minimization problem (1.2) requires first a thorough analysis of the state system. In this section we answer the question of whether states $y=(v, \vartheta, \Phi)$ exist for any given control $u$ of the state system (1.1). Since we have a nonlinear coupled problem, we will first derive results for each part, and then use a fixed point argument to show existence of a solution. As with most nonlinear problems, we show that under certain regularity assumptions on the states, we obtain uniqueness via a Gronwall argument, which then guarantees the well-posedness of the control-to-state map. We also derive necessary bounds for the states required to show existence of minimizers for the minimization problem (1.2). We state the main result for this section.

THEOREM 2.1. Under assumptions 1-2 given below, we obtain for any given control function $u \in \mathcal{U}_{a d} \hookrightarrow L^{\infty}(\Sigma)$ a unique state $y \in \mathcal{X}_{\infty}$ of the system (1.1) with the state space $\mathcal{X}_{\infty}$ defined in Section 2.5.

Before we begin the proof of the theorem, we collect all the required estimates obtained for each subproblem.

2.1. Navier-Stokes equation. Here, we will use the solenoidal spaces $\mathcal{H}, \mathcal{H}^{1}$ and their corresponding spaces $\mathcal{V}, \mathcal{W}(\mathcal{V})$. For the flow of the fluid, we consider the incompressible viscous Navier-Stokes equations for the velocity field $v$ and pressure $p$ given by

$$
\begin{aligned}
\nabla \cdot v & =0, & & \text { in } Q, \\
\partial_{t} v-\operatorname{Pr} \nabla \cdot \nu \nabla v+v \cdot \nabla v+\nabla p-\operatorname{RaPr} \vartheta e_{2} & =f_{v}, & & \text { in } Q, \\
v & =0, & & \text { on } \Sigma, \\
v(0) & =v_{0}, & & \text { in } \Omega,
\end{aligned}
$$

where $\vartheta, f_{v}$, and $v_{0}$ are given functions and vector fields respectively. We call $v$ a weak solution of (2.1) if it fulfills the following weak formulation:

$$
v \in \mathcal{W}(\mathcal{V}):\left\{\begin{array}{r}
\left\langle\partial_{t} v+A_{v}(v)+B_{v}(v, v)-F_{v}(\vartheta), \varphi_{1}\right\rangle=0 \\
\left(v(0)-v_{0}, \varphi_{2}\right)_{\Omega}=0
\end{array}\right\} \forall \varphi \in \mathcal{V} \times \mathcal{H}
$$

where the operators $A_{v}, B_{v}$, and $F_{v}$ are are induced by the forms

$$
\left\langle A_{v}(v), \varphi_{1}\right\rangle=\sum_{i}\left(\operatorname{Pr} \nu \nabla v_{i}, \nabla \varphi_{1, i}\right)_{Q}
$$




$$
\begin{aligned}
\left\langle B_{v}(v, w), \varphi_{1}\right\rangle & =\left\langle v \cdot \nabla w, \varphi_{1}\right\rangle, \\
\left\langle F_{v}(\vartheta), \varphi_{1}\right\rangle & =\left(\operatorname{RaPr} \vartheta e_{2}+f_{v}, \varphi_{1}\right)_{Q},
\end{aligned}
$$

for $v, w \in \mathcal{W}(\mathcal{V})$. For simplicity, we use the notation $B_{v}(v)=B_{v}(v, v)$ for all $v \in \mathcal{W}(\mathcal{V})$.

Observe that, due to the introduction of the solenoidal spaces $\mathcal{H}, \mathcal{H}^{1}$ along with the weak formulation by Leray, the pressure term $p$ falls out of the weak formulation. This gives us the possibility to concentrate on the existence and uniqueness of the velocity field $v$ alone, and later use this solution to retrieve the corresponding pressure $p$ with the help of Helmholtz-Weyl's decomposition, or more generally the De Rham theory (c.f. [44]). Since existence and uniqueness for the incompressible viscous Navier-Stokes equation with no-slip boundary are well-known, we may recall results from the standard literature, e.g., [28, 44]. We collect the necessary results pertaining to the Navier-Stokes equation in the following theorem.

TheOREM 2.2. Let $F_{v}(\vartheta) \in \mathcal{V}^{*}$ and $v_{0} \in \mathcal{H}$, then there exists a unique solution $v \in$ $\mathcal{W}(\mathcal{V})$ to (2.2) satisfying the energy inequality

$$
\|v\|_{\mathcal{W}(\mathcal{V})} \leq c\left(\left\|F_{v}(\vartheta)\right\|_{\mathcal{V}^{*}}+\left\|F_{v}(\vartheta)\right\|_{\mathcal{V}^{*}}^{2}+\left\|v_{0}\right\|_{\mathcal{H}}+\left\|v_{0}\right\|_{\mathcal{H}}^{2}\right),
$$

with constant $c>0$. The solution $v$ also belongs to $\left[L^{4}(Q)\right]^{2}$ and satisfies the estimate

$$
\|v\|_{4, Q} \leq c_{v}\left(\left\|F_{v}(\vartheta)\right\|_{\mathcal{V}^{*}}+\left\|v_{0}\right\|_{\mathcal{H}}\right)
$$

with constant $c_{v}>0$ independent of $v, \vartheta, f_{v}$, and $v_{0}$.

Furthermore, there exists a unique $p \in \mathcal{C}\left(\bar{I} ; L_{0}^{2}(\Omega)\right)$, such that the pair $(v, p)$ fulfills (2.1) in the distributional sense, where $L_{0}^{2}(\Omega) \subset L^{2}(\Omega)$ denotes the subspace consisting of elements orthogonal to the constants.

Proof. The first assertion is a consequence of [44, Theorem III.3.1] together with [44, Theorem III.3.2]. That the solution $v$ belongs to $\left[L^{4}(Q)\right]^{2}$ follows directly from the fact that $v \in \mathcal{C}(\bar{I} ; \mathcal{H})$ (c.f. [44, Lemma III.1.2]) and the inequality

$$
\|v\|_{4, Q} \leq 2^{1 / 4}\|v\|_{2, \infty, Q}^{1 / 2}\|\nabla v\|_{2, Q}^{1 / 2} \quad \forall v \in \mathcal{V} \cap L^{\infty}(\bar{I} ; \mathcal{H}),
$$

which results from [44, Lemma III.3.3]. The estimate follows from

$$
\frac{1}{2} \frac{\mathrm{d}}{\mathrm{d} t}\|v(t)\|_{2, \Omega}^{2}+\left(\operatorname{Pr} \underline{\nu}-\frac{\delta}{2}\right)\|v(t)\|_{\mathcal{H}^{1}}^{2} \leq \frac{1}{2 \delta}\left\|F_{v}[\vartheta](t)\right\|_{\mathcal{H}^{-1}}^{2},
$$

which is obtained by testing (2.2) with $\varphi_{1}=v$ and an application of Young's inequality with $\delta<2 \operatorname{Pr} \underline{\nu}$. Here, we also used the equivalent norm $\|w\|_{\mathcal{H}^{1}}^{2}=\sum_{i}\left\|\nabla w_{i}\right\|_{2, \Omega}^{2}$. By integrating up to $t \in I$ and multiplying by 2 , we obtain

$$
\|v(t)\|_{2, \Omega}^{2} \leq \frac{1}{\delta}\left\|F_{v}(\vartheta)\right\|_{\mathcal{V}^{*}}^{2}+\left\|v_{0}\right\|_{2, \Omega}^{2},
$$

which holds for a.e. $t \in I$, and thus

$$
\|v\|_{2, \infty, Q}^{2}=\operatorname{esssup}\|v(t)\|_{2, \Omega}^{2} \leq \frac{1}{\delta}\left\|F_{v}[\vartheta]\right\|_{\mathcal{V}^{*}}^{2}+\left\|v_{0}\right\|_{2, \Omega}^{2} .
$$

Moreover, by integrating up to $t=T$, we obtain

$$
\|v\|_{\mathcal{V}}^{2} \leq c_{1}\left(\frac{1}{\delta}\left\|F_{v}(\vartheta)\right\|_{\mathcal{V}^{*}}^{2}+\left\|v_{0}\right\|_{2, \Omega}^{2}\right),
$$


with $c_{1}=(2 \operatorname{Pr} \underline{\nu}-\delta)^{-1}$. Finally, from the estimate given in (2.3), we find that

$$
\|v\|_{4, Q}^{2} \leq 2^{1 / 2}\|v\|_{2, \infty, Q}\|\nabla v\|_{2, Q} \leq 2^{1 / 2} c_{1}\left(\frac{1}{\delta}\left\|F_{v}(\vartheta)\right\|_{\mathcal{V}^{*}}^{2}+\left\|v_{0}\right\|_{2, \Omega}^{2}\right),
$$

and hence the required estimate by the equivalence of norms in $\mathbb{R}^{n}$.

For the second assertion we refer the reader to the discussion made at the end of [44, Section III. 3.5], which is a consequence of a pointwise (in time) argument with the Helmholtz-Weyl decomposition or the result of De Rham (c.f. [44, Proposition I.1.1]), and the isomorphism of the gradient operator from $L_{0}^{2}(\Omega)$ into $H^{-1}(\Omega)$ due to [44, Remark I.1.4].

Remark 2.1. It is easy to see that $B_{v}: \mathcal{W}(\mathcal{V}) \rightarrow \mathcal{V}^{*}$ is continuously Frechét differentiable with derivative

$$
\left\langle\mathrm{d} B_{v}(v)[w], \varphi\right\rangle=\langle w \cdot \nabla v+v \cdot \nabla w, \varphi\rangle \quad \forall \varphi \in \mathcal{V},
$$

and any $v, w \in \mathcal{W}(\mathcal{V})$.

If $F_{v}(\vartheta)$ and $v_{0}$ are further essentially bounded, we have the following result.

Theorem 2.3. If $F_{v}(\vartheta) \in\left[L^{\infty}(Q)\right]^{2}$ and $v_{0} \in \mathcal{H} \cap\left[L^{\infty}(\Omega)\right]^{2}$, then there exists a unique solution $v \in \mathcal{W}(\mathcal{V}) \cap\left[L^{\infty}(Q)\right]^{2}$ to (2.2) fulfilling the bounds

$$
\underline{q}_{v} \leq v \leq \bar{q}_{v}
$$

with $\underline{q}_{v}=\min \left\{\underline{v}_{0}, \underline{F}_{v}(\vartheta), 0\right\}$ and $\bar{q}_{v}=e^{T} \max \left\{\bar{v}_{0}, \bar{F}_{v}(\vartheta), 0\right\}$.

Proof. Since the embeddings $\left[L^{\infty}(Q)\right]^{2} \hookrightarrow \mathcal{V}^{*}$ and $\mathcal{H} \cap\left[L^{\infty}(\Omega)\right]^{2} \hookrightarrow \mathcal{H}$ are continuous, we have from Theorem 2.2 that $v \in \mathcal{W}(\mathcal{V})$. We define

$$
\underline{w}:=\min \left\{\underline{v}_{0}, \underline{F}_{v}(\vartheta), 0\right\} \quad \text { and } \quad \bar{w}:=\max \left\{\bar{v}_{0}, \bar{F}_{v}(\vartheta), 0\right\} .
$$

Define $w:=e^{-t} v$. Substituting $v=e^{t} w$ into (2.2) and dividing by $e^{t}$, we obtain

$$
w \in \mathcal{W}(\mathcal{V}):\left\{\begin{array}{r}
\left\langle\partial_{t} w+A_{v}(w)+e^{t} B_{v}(w)+w-e^{-t} F_{v}(\vartheta), \varphi_{1}\right\rangle=0 \\
\left(w(0)-v_{0}, \varphi_{2}\right)_{\Omega}=0
\end{array}\right\} \forall \varphi \in \mathcal{V} \times \mathcal{H}
$$

Now consider the set $\chi^{+}=\{(x, t) \in Q \mid w(x, t)>\bar{w}\}$. Testing (2.4) with $\varphi^{+}:=(w-\bar{w})^{+}$ yields, for almost every $t \in I$, the estimate

$$
\frac{1}{2}\left\|\varphi^{+}(t)\right\|_{2, \Omega}^{2}+\int_{0}^{t}\left\|\varphi^{+}(\tau)\right\|_{2, \Omega}^{2} \mathrm{~d} \tau \leq-\left(\bar{q}_{v}-e^{-t} F_{v}(\vartheta), \varphi^{+}\right)_{Q} \leq 0
$$

which tells us that $\chi^{+}$is necessarily empty, i.e., $\chi^{+}=\emptyset$. Here we used the fact that

$$
\left\langle A_{v}\left(\varphi^{+}\right), \varphi^{+}\right\rangle \geq 0 \text {. }
$$

Similarly, testing (2.4) with $\varphi^{-}:=(w-\underline{w})^{-}$shows that $\chi^{-}=\{(x, t) \in Q \mid w(x, t)<\underline{w}\}$ is empty. Thus we find that

$$
\underline{w} \leq w \leq \bar{w} \quad \Rightarrow \quad \underline{q}_{v}=e^{0} \underline{w} \leq v \leq e^{T} \bar{w}=\bar{q}_{v},
$$

and thus $\|v\|_{\infty, Q} \leq M_{v}:=\max \left\{\left|\underline{q}_{v}\right|,\left|\bar{q}_{v}\right|\right\}$, which concludes the proof. 
To conclude this section, we recall a regularity result obtained by Ladyzhenskaya for the solutions for given data with better regularity, which can be found in [27, Theorem 3] and the references therein.

Proposition 2.2. If $F_{v}(\vartheta) \in\left[L^{2}(Q)\right]^{2}, \sqrt{t} \partial_{t} F_{v}(\vartheta) \in \mathcal{V}^{*}$, and $v_{0} \in \mathcal{H}^{1}$, then the solution $v$ to (2.2) has the properties

$$
v \in \mathcal{C}\left(\bar{I} ; \mathcal{H}^{1}\right) \quad \text { and } \quad \partial_{t} v \in L^{2}(I ; \mathcal{H}) \cap L^{\infty}([\delta, T], \mathcal{H}) \cap L^{2}\left((\delta, T) ; \mathcal{H}^{1}\right),
$$

for any $\delta \in I$.

2.2. Nonlinearity. In this section we will deduce some properties for the scaled Planck's function for a black body $I_{b}$ defined by

$$
I_{b}: G \times \mathbb{R} \rightarrow \mathbb{R}: \quad I_{b}(x, w)=(w+1)^{4},
$$

where $G$ denotes either $\Omega, \Gamma, Q$, or $\Sigma$. More precisely, we will show the following.

Lemma 2.4. The induced Nemytskij-operator $I_{b}: L^{\infty}(G) \rightarrow L^{\infty}(G)$ defined above is continuously Fréchet differentiable and for $w, \varphi \in L^{\infty}(G)$,

$$
\left(\mathrm{d} I_{b}(w)[\varphi]\right)(x)=4(w(x)+1)^{3} \varphi(x) \quad \text { for a.e. } x \in G .
$$

Furthermore, there exists Lipschitz-constants $l_{j}(M), j=0,1$, such that the following estimates hold true for all $p \in[1, \infty]$ :

$$
\left\|\mathrm{d}^{j} I_{b}\left(w_{1}\right)-\mathrm{d}^{j} I_{b}\left(w_{2}\right)\right\|_{p, G} \leq l_{j}(M)\left\|w_{1}-w_{2}\right\|_{p, G}, \quad j \in\{0,1\},
$$

for all $w_{i} \in L^{\infty}(G)$ with $\left\|w_{i}\right\|_{\infty, G} \leq M$.

Proof. The scaled Planck function $I_{b}$ clearly satisfies the Carathéodory conditions since $(w+1)^{4}$ is continuous in $\mathbb{R}$. Furthermore, it fulfills the following conditions:

Boundedness condition. There exists bounds $0<K_{j}<\infty$ such that

$$
\left|\partial_{w}^{j} I_{b}(x, 0)\right| \leq K_{j} \quad \text { for a.e. } x \in G .
$$

Local Lipschitz condition. There exist Lipschitz constants $l_{j}=l_{j}(M)$, such that

$$
\left|\partial_{w}^{j} I_{b}\left(x, w_{1}\right)-\partial_{w}^{j} I_{b}\left(x, w_{2}\right)\right| \leq l_{j}(M)\left|w_{1}-w_{2}\right|,
$$

for all $w_{i} \in \mathbb{R}$ with $\left|w_{i}\right| \leq M$.

The assertion follows from [47, Lemmas 4.11, 4.12, and 4.13], where

$$
I_{b}(w)(x)=I_{b}(x, w(x))=(w(x)+1)^{4} \quad \text { for a.e. } x \in G
$$

is the induced Nemytskij-operator for all $w \in L^{\infty}(G)$.

Note that we use the same notation for a function and its Nemytskij-operator. This ambiguity should not cause any confusion once we mention the spaces involved.

Lemma 2.5. If $\left\{w_{k}\right\}_{k} \subset L^{\infty}(G)$ be a bounded sequence that is weakly-* convergent to an element $w_{*}$, i.e., $w_{k} \rightarrow^{*} w_{*}$ in $L^{\infty}(G)$ and almost everywhere, i.e., $w_{k}(x) \rightarrow w_{*}(x)$ a.e. $x \in G$, then

$$
I_{b}\left(w_{k}\right) \rightarrow I_{b}\left(w_{*}\right) \text { in } L^{p}(G)
$$


for any $p \in(1, \infty)$.

Proof. Let $\left\{w_{k}\right\}_{k} \subset L^{\infty}(G)$ be bounded. Then $\left\{I_{b}\left(w_{k}\right)\right\}_{k}$ is bounded in $L^{p}(G)$, $p \in(1, \infty)$ due to Lemma 2.4. This gives us the existence of a weakly convergent subsequence, denoted again by $\left\{I_{b}\left(w_{k}\right)\right\}_{k}$, with the weak limit $I_{b, p}^{*} \in L^{p}(G)$ for each $p \in(1, \infty)$, i.e., $I_{b}\left(w_{k}\right) \rightarrow I_{b, p}^{*}$ in $L^{p}(G)$. Since $w_{k}(x) \rightarrow w_{*}(x)$ a.e. $x \in G$, we have, due the continuity of the Planck function, that

$$
I_{b}\left(w_{k}\right)(x)=I_{b}\left(x, w_{k}(x)\right) \rightarrow I_{b}\left(x, w_{*}(x)\right)=I_{b}\left(w_{*}\right)(x) \quad \text { for a.e. } x \in G .
$$

We complete the proof by referring to [43, Lemma 3.9], which allows one to identify the weak limits, i.e., $I_{b, p}^{*} \equiv I_{b}\left(w_{*}\right)$ for all $p \in(1, \infty)$.

2.3. $\mathrm{SP}_{3}$-approximation. Recall the $\mathrm{SP}_{3}$-approximation for the incident radiation $\phi$ in a given domain $\Omega$ given by the system

$$
\begin{aligned}
-\nabla \cdot \frac{\epsilon^{2}}{3 \sigma_{t}}(\nabla \phi+2 \nabla \xi)+\sigma_{a} \phi & =\sigma_{a} I_{b}(\vartheta), \\
-\nabla \cdot \frac{\epsilon^{2}}{3 \sigma_{t}}\left(\frac{2}{5} \nabla \phi+\frac{11}{7} \nabla \xi\right)+\sigma_{t} \xi & =0,
\end{aligned}
$$

in $\Omega$,

with boundary conditions

$$
\begin{aligned}
n \cdot \frac{\epsilon^{2}}{3 \sigma_{t}}(\nabla \phi+2 \nabla \xi)+\alpha_{1,1} \phi+\alpha_{1,2} \xi & =\alpha_{1,1} I_{b}(u), \\
n \cdot \frac{\epsilon^{2}}{3 \sigma_{t}}\left(\frac{2}{5} \nabla \phi+\frac{11}{7} \nabla \xi\right)+\alpha_{2,1} \phi+\alpha_{2,2} \xi & =\alpha_{2,1} I_{b}(u),
\end{aligned}
$$

where the extinction, absorption, and scattering coefficients are denoted by $\sigma_{t}, \sigma_{a}$, and $\sigma_{s}$ respectively, and the coefficients $\alpha_{i, j}$ depend on the reflectivity of the material on the surface $\Gamma$. The extinction coefficient $\sigma_{t}$ is given by the sum of the absorption and scattering coefficients, i.e., $\sigma_{t}=\sigma_{a}+\sigma_{s}$.

We call $\Phi=\left(\Phi_{1}, \Phi_{2}\right)^{\mathrm{T}}=(\phi, \xi)^{\mathrm{T}}$ a weak solution of (2.5) if it fulfills

$$
\Phi \in\left[H^{1}(\Omega)\right]^{2}:\left\langle A_{\Phi}(\Phi)+B_{\Phi}(\Phi)-F_{\Phi}(u, \vartheta), \varphi\right\rangle=0 \quad \forall \varphi \in\left[H^{1}(\Omega)\right]^{2},
$$

where the operators $A_{\Phi}, B_{\Phi}, F_{\Phi}$ are induced by the forms

$$
\begin{aligned}
\left\langle A_{\Phi}(\Phi), \varphi\right\rangle & =\sum_{i, j}\left(\frac{\epsilon^{2}}{3 \sigma_{t}} \hat{\mathrm{a}}_{i, j} \nabla \Phi_{j}\right)_{\Omega} \nabla \varphi_{i}+(\hat{\alpha} \Phi, \varphi)_{\Gamma}, \\
\left\langle B_{\Phi}(\Phi), \varphi\right\rangle & =(\hat{\sigma} \Phi, \varphi)_{\Omega}, \\
\left\langle F_{\Phi}(u, \vartheta), \varphi\right\rangle & =\left(\sigma_{a} I_{b}(\vartheta) e_{1}, \varphi\right)_{\Omega}+\left(\hat{\alpha}_{, 1} I_{b}(u), \varphi\right)_{\Gamma},
\end{aligned}
$$

with the matrices and vector given, respectively, by

$$
\hat{\mathrm{a}}=\left(\begin{array}{cc}
1 & 2 \\
\frac{2}{5} & \frac{11}{7}
\end{array}\right), \quad \hat{\sigma}=\left(\begin{array}{cc}
\sigma_{a} & 0 \\
0 & \sigma_{t}
\end{array}\right), \quad \hat{\alpha}=\left(\begin{array}{cc}
\alpha_{1,1} & \alpha_{1,2} \\
\alpha_{2,1} & \alpha_{2,2}
\end{array}\right), \quad \hat{\alpha}_{, 1}=\left(\begin{array}{c}
\alpha_{1,1} \\
\alpha_{2,1}
\end{array}\right) .
$$

Under a proper assumption on the boundary matrix $\hat{\alpha}$ we obtain existence and uniqueness for (2.6).

THEOREM 2.6. Let $\hat{\alpha}$ be semi-positive definite and $F_{\Phi}(u, \vartheta) \in\left[H^{1}(\Omega)^{*}\right]^{2}$. Then there exists a unique solution $\Phi \in\left[H^{1}(\Omega)\right]^{2}$ fulfilling (2.6) such that the estimate

$$
\|\Phi\|_{\left[H^{1}(\Omega)\right]^{2}} \leq c_{\Phi}\left\|F_{\Phi}(u, \vartheta)\right\|_{\left[H^{1}(\Omega)^{*}\right]^{2}}
$$


holds true with constant $c_{\Phi}>0$ not depending on $\Phi$.

Proof. We will apply the theorem of Lax-Milgram to obtain the assertion. The continuity of the operator $A_{\Phi}+B_{\Phi}:\left[H^{1}(\Omega)\right]^{2} \rightarrow\left[H^{1}(\Omega)^{*}\right]^{2}$ is clear. All that is left to show is its coercivity.

Consider the scalar product corresponding to the symmetric part $\hat{a}^{\mathrm{sym}}$ of $\hat{a}$. One may easily show that $\hat{a}^{\text {sym }}$ is positive definite and hence the eigenvalue decomposition of $\hat{a}^{\mathrm{sym}}$ may be given by $\hat{\mathrm{a}}^{\mathrm{sym}}=\hat{\mathrm{u}} \hat{\mathrm{s}} \hat{\mathrm{u}}^{\mathrm{T}}$, where $\hat{\mathrm{u}}$ is an orthogonal matrix and $\hat{\mathrm{s}}$ is a diagonal matrix with positive eigenvalues. We thus have

$$
\begin{aligned}
\sum_{i, j}\left(\frac{\epsilon^{2}}{3 \sigma_{t}} \hat{\mathrm{a}}_{i, j}^{\mathrm{sym}} \nabla \Phi_{j}, \nabla \Phi_{i}\right)_{\Omega} & =\sum_{i}\left(\frac{\epsilon^{2}}{3 \sigma_{t}} \hat{\mathrm{s}}_{i, i} \sum_{j} \hat{\mathrm{u}}_{i, j}^{\mathrm{T}} \nabla \Phi_{j}, \sum_{j} \hat{\mathrm{u}}_{i, j}^{\mathrm{T}} \nabla \Phi_{j}\right)_{\Omega} \\
& \geq \min \left\{s_{1,1}, s_{2,2}\right\} \frac{\epsilon^{2}}{3 \overline{\sigma_{t}}}\left\|\sum_{j} \hat{\mathrm{u}}_{i, j}^{\mathrm{T}} \nabla \Phi_{j}\right\|_{2, \Omega}^{2}=c_{1}\|\nabla \Phi\|_{2, \Omega}^{2},
\end{aligned}
$$

where the last equality follows from the orthogonality of $\hat{\mathrm{u}}^{\mathrm{T}}$. Altogether have

$$
\sum_{i, j}\left(\frac{\epsilon^{2}}{3 \sigma_{t}} \hat{\mathrm{a}}_{i, j} \nabla \Phi_{j}, \nabla \Phi_{i}\right)_{\Omega}=\sum_{i, j}\left(\frac{\epsilon^{2}}{3 \sigma_{t}}\left(\hat{\mathrm{a}}_{i, j}^{\text {sym }}+\hat{\mathrm{a}}_{i, j}^{\text {skew }}\right) \nabla \Phi_{j}, \nabla \Phi_{i}\right)_{\Omega} \geq c_{1}\|\nabla \Phi\|_{2, \Omega}^{2},
$$

since the scalar product of the skew-symmetric part $\sum_{i, j}\left(\hat{a}_{i, j}^{\text {skew }} \nabla \Phi_{j}, \nabla \Phi_{i}\right)_{\Omega}=0$. Since $\hat{\alpha}$ is semi-positive definite, we have, for all $\Phi \in\left[H^{1}(\Omega)\right]^{2} \hookrightarrow\left[L^{2}(\Gamma)\right]^{2}$,

$$
\Phi^{\mathrm{T}} \hat{\alpha} \Phi \geq 0 \quad \text { a.e. on } \Gamma \text {, }
$$

and thus $(\hat{\alpha} \Phi, \Phi)_{\Gamma} \geq 0$ due to the monotonicity of integrals. Finally we obtain the coercivity for $A_{\Phi}+B_{\Phi}$, i.e.,

$$
\left\langle\left(A_{\Phi}+B_{\Phi}\right)(\Phi), \Phi\right\rangle \geq c_{1}\|\nabla \Phi\|_{2, \Omega}^{2}+c_{2}\|\Phi\|_{2, \Omega}^{2} \geq c_{\Phi}\|\Phi\|_{\left[H^{1}(\Omega)\right]^{2}}^{2},
$$

where $c_{2}=\min \left\{\underline{\sigma}_{a}, \underline{\sigma}_{t}\right\}$ and $c_{\Phi}=\min \left\{c_{1}, c_{2}\right\}$. We conclude the theorem by applying the theorem of Lax-Milgram (cf. [42]).

At this point, one might think that $H^{1}(\Omega)$ solutions are sufficient for the analysis of the state system. This is unfortunately not the case due to the lack of monotonicity of the full system. We will require uniform bounds on the incident radiation $\phi$ for data that are uniformly bounded; in other words, a maximum principle for (2.5). However, the presence of cross-diffusion and boundary coupling make the elliptic system noncooperative. An exposition on cooperative elliptic systems can be found, for example, in [6], where the proofs depend on properties of the principal eigenvalues of the elliptic system and the Krein-Rutman Theorem on Banach lattices. Due to the lack of these tools for non-cooperative systems, work has to be done using comparison principles via sub- and supersolutions (c.f. [11]) to show a maximum principle for (2.5).

Following the works of E. W. Larsen et al. in [30,31], we obtain an equivalent system by a similarity transformation of (2.5). More precisely, the equivalent system is obtained via a diagonalization procedure for â with the new variables $\psi=\hat{\mathrm{T}} \Phi$, where

$$
\hat{\mathrm{T}}=\left(\begin{array}{cc}
1 & \lambda_{1} \\
1 & \lambda_{2}
\end{array}\right), \quad \lambda_{i}=\frac{5}{7}\left(1+(-1)^{i} 3 \sqrt{\frac{6}{5}}\right), i=1,2 .
$$

By multiplying (2.5) with $\hat{\mathrm{T}}$ from the left and using the inverse transformation $\hat{\mathrm{T}}^{-1}$, we obtain an equivalent system

$$
-\nabla \cdot \epsilon^{2} \frac{\mu_{i}^{2}}{\sigma_{t}} \nabla \psi_{i}+\sigma_{t} \psi_{i}=\sigma_{s} \sum_{j} \omega_{j} \psi_{j}+\sigma_{a} I_{b}(\vartheta) \quad \text { in } \Omega,
$$


with boundary conditions

$$
n \cdot \epsilon^{2} \frac{\mu_{i}^{2}}{\sigma_{t}} \nabla \psi_{i}+\beta_{i, i} \psi_{i}+\beta_{i, j} \psi_{j}=\eta_{i} I_{b}(u) \quad \text { on } \Gamma,
$$

where the constants $\mu_{i}$ and $\omega_{i}$ are given respectively by

$$
\mu_{i}^{2}=\frac{1}{7}\left(3+2(-1)^{i} \sqrt{\frac{6}{5}}\right) \quad \text { and } \quad \omega_{i}=\frac{1}{2}+\frac{(-1)^{i-1}}{6} \sqrt{\frac{5}{6}} .
$$

The coefficients $\beta_{i, j}$ are related to the $\alpha_{i, j}$ by $\hat{\beta}=\hat{\mathrm{T}} \hat{\alpha} \hat{\mathrm{T}}^{-1}$. Furthermore, the coefficients $\eta_{i}$ have the form $\eta_{i}=\sum_{j} \beta_{i, j}$. We note that the incident radiation $\phi$ can be given as a linear combination of the dummy variables $\psi_{i}$, namely

$$
\phi=\omega_{1} \psi_{1}+\omega_{2} \psi_{2},
$$

where the $\omega_{i}$ are as given above with $\sum_{i} \omega_{i}=1$.

Similar to (2.6), we call $\psi$ a weak solution of (2.7) if it fulfills the weak formulation

$$
\psi \in\left[H^{1}(\Omega)\right]^{2}:\left\langle A_{\psi}(\psi)+B_{\psi}(\psi)-F_{\psi}(u, \vartheta), \varphi\right\rangle=0 \quad \forall \varphi \in\left[H^{1}(\Omega)\right]^{2},
$$

where the operators $A_{\psi}, B_{\psi}, F_{\psi}$ are induced by the forms

$$
\begin{aligned}
\left\langle A_{\psi, i}(\psi), \varphi_{i}\right\rangle & =\left(\epsilon^{2} \frac{\mu_{i}^{2}}{\sigma_{t}} \nabla \psi_{i}, \nabla \varphi_{i}\right)_{\Omega}+\left(\beta_{i, i} \psi_{i}+\beta_{i, j} \psi_{j}, \varphi_{i}\right)_{\Gamma}, \\
\left\langle B_{\psi, i}(\psi), \varphi_{i}\right\rangle & =\left(\left(\sigma_{t}-\sigma_{s} \omega_{i}\right) \psi_{i}-\sigma_{s} \omega_{j} \psi_{j}, \varphi_{i}\right)_{\Omega}, \\
\left\langle F_{\psi, i}(u, \vartheta), \varphi_{i}\right\rangle & =\left(\sigma_{a} I_{b}(\vartheta), \varphi_{i}\right)_{\Omega}+\left(\eta_{i} I_{b}(u), \varphi_{i}\right)_{\Gamma} .
\end{aligned}
$$

EXAMPLE 2.3. In the case of no reflection $(\rho=0)$ on the boundary $\Gamma$ (cf. [31]),

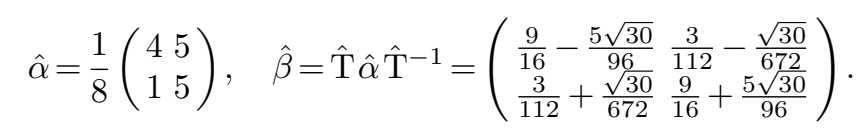

Under the following assumption on the transformed boundary matrix $\hat{\beta}$ we obtain existence and uniqueness of solutions for $(2.8)$ in $\left[H^{1}(\Omega) \cap \mathcal{C}(\bar{\Omega})\right]^{2}$ that further fulfill uniform bounds for uniformly bounded data.

Assumption 1. $\hat{\beta}$ is strictly diagonally dominant with positive diagonal entries.

REMARK 2.4. Assumption 1 poses no restriction for all physical related reflectivity $\rho$ of the material on the boundary $\Gamma$. Example 2.3 clearly shows that $\hat{\beta}$ is strictly diagonally dominant with positive diagonal entries and thus also positive definite, which leads to the positive definiteness of $\hat{\alpha}$.

Theorem 2.7. Let Assumption 1 hold, $\vartheta \in L^{\infty}(\Omega)$, and $u \in L^{\infty}(\Gamma)$. Then there exists a unique solution $\psi \in\left[H^{1}(\Omega) \cap \mathcal{C}(\bar{\Omega})\right]^{2}$ fulfilling (2.8) such that

$$
I_{b}(\underline{q}) \leq \psi_{i} \leq I_{b}(\bar{q})
$$

with $\underline{q}=\min \{\underline{\vartheta}, \underline{u}, 0\}$ and $\bar{q}=\max \{\bar{\vartheta}, \bar{u}, 0\}$.

Proof. Existence. Let $(w, \tau) \in[\mathcal{C}(\bar{\Omega})]^{2} \times[0,1]$. Consider the auxiliary problem

$$
\psi \in V: \quad\left\langle\left(\tilde{A}_{\psi}+\tilde{B}_{\psi}\right)[\psi, w, \tau]-\tau F_{\psi}[u, \vartheta], \varphi\right\rangle=0 \quad \forall \varphi \in\left[H^{1}(\Omega)\right]^{2},
$$


where the operators $\tilde{A}_{\psi}, \tilde{B}_{\psi}$, are induced by the forms

$$
\begin{aligned}
& \left\langle\tilde{A}_{\psi, i}(\psi, w, \tau), \varphi_{i}\right\rangle=\left(\epsilon^{2} \frac{\mu_{i}^{2}}{\sigma_{t}} \nabla \psi_{i}, \nabla \varphi_{i}\right)_{\Omega}+\tau\left(\beta_{i, i} \psi_{i}+\beta_{i, j} \zeta\left(\psi_{i}, w_{j}\right), \varphi_{i}\right)_{\Gamma} \\
& \left\langle\tilde{B}_{\psi, i}(\psi, w, \tau), \varphi_{i}\right\rangle=\left(\sigma_{t} \psi_{i}-\tau \sigma_{s}\left(\omega_{i} \psi_{i}+\omega_{j} \zeta\left(\psi_{i}, w_{j}\right)\right), \varphi_{i}\right)_{\Omega}
\end{aligned}
$$

with $\zeta: L^{p}(G) \times \mathcal{C}(\bar{\Omega}) \rightarrow L^{p}(G), p \in[1, \infty), \tau \in[0,1]$, for $G=\Omega, \Gamma$, given by

$$
\zeta\left(\psi_{i}, w_{j}\right)=\left\{\begin{aligned}
\psi_{i}, & \psi_{i}<I_{b}(\underline{q}), \\
\varrho\left(w_{j}\right), & I_{b}(\underline{q}) \leq \psi_{i} \leq I_{b}(\bar{q}), \\
\psi_{i}, & \psi_{i}>I_{b}(\bar{q}),
\end{aligned}\right.
$$

where $\varrho: \mathcal{C}(\bar{\Omega}) \rightarrow \mathcal{C}(\bar{\Omega})$ is the cutoff operator defined by

$$
\varrho\left(w_{j}\right)=\max \left\{I_{b}(\underline{q}), \min \left\{w_{j}, I_{b}(\bar{q})\right\}\right\} .
$$

We will show that the operator

$$
L_{i}:=\tilde{A}_{\psi, i}\left(\cdot, w_{j}, \tau\right)+\tilde{B}_{\psi, i}\left(\cdot, w_{j}, \tau\right): H^{1}(\Omega) \rightarrow H^{1}(\Omega)^{*}
$$

is hemicontinuous, strictly monotone, and coercive. Hemicontinuity of $L_{i}$ can easily be seen due to its boundedness. To show strict monotonicity of $L_{i}$, it is sufficient to show that the operator $\zeta(\cdot, w)$ is monotone, i.e.,

$$
\left\langle\zeta\left(\psi_{i}^{1}, w\right)-\zeta\left(\psi_{i}^{2}, w\right), \psi_{i}^{1}-\psi_{i}^{2}\right\rangle \geq 0,
$$

for any $w \in \mathcal{C}(\bar{\Omega})$ and $\psi_{i}^{1}, \psi_{i}^{2} \in L^{p}(G)$. We consider several cases that may occur. Let $\chi^{+}(G)$ be the set of points in $G$ such that $\psi_{i}^{1}>\psi_{i}^{2}$. For the case where $\psi_{i}^{1} \geq I_{b}(\bar{q})$ and $I_{b}(\underline{q}) \leq \psi_{i}^{2} \leq I_{b}(\bar{q})$, we have, due to the cutoff operator, that $\psi_{i}^{1} \geq \varrho(w)$. For the case where $I_{b}(\underline{q}) \leq \psi_{i}^{1} \leq I_{b}(\bar{q})$ and $\psi_{i}^{2} \leq I_{b}(\underline{q})$, we have, again due to the the cutoff operator, that $\varrho(w) \geq \psi_{i}^{2}$. When $I_{b}(\underline{q}) \leq \psi_{i}^{1}, \psi_{i}^{2} \leq I_{b}(\bar{q})$, then we have $\zeta\left(\psi_{i}^{1}, w\right)-\zeta\left(\psi_{i}^{2}, w\right)=0$. Arguments for the set $\chi^{-}(G)=\left\{x \in G \mid \psi_{i}^{1}<\psi_{i}^{2}\right\}$ can be made analogously. We conclude the strict monotonicity of the operator $L_{i}$ from the strict monotonicity of its linear parts. Due to Assumption 1 and the fact that $\sigma_{t}=\sigma_{a}+\sigma_{s}$ and $\sum_{i} \omega_{i}=1$, we have

$$
\begin{gathered}
\left\langle L_{i} \psi_{i}, \psi_{i}\right\rangle \geq \epsilon^{2} \frac{\mu_{i}^{2}}{\overline{\sigma_{t}}}\left\|\nabla \psi_{i}\right\|_{2, \Omega}^{2}+\tau\left(\beta_{i, i}-\left|\beta_{i, j}\right|\right)\left\|\psi_{i}\right\|_{2, \Gamma}^{2}-\tau\left|\beta_{i, j}\right|\left\|w_{j}\right\|_{2, \Gamma}\left\|\psi_{i}\right\|_{2, \Gamma} \\
\quad+\left(\underline{\sigma_{a}}+(1-\tau) \underline{\sigma_{s}}\right)\left\|\psi_{i}\right\|_{2, \Omega}^{2}-\tau \overline{\sigma_{s}} \omega_{j}\left\|w_{j}\right\|_{2, \Omega}\left\|\psi_{i}\right\|_{2, \Omega}, \\
\geq c_{1}\left\|\psi_{i}\right\|_{H^{1}(\Omega)}^{2}-c_{2} \tau\left\|w_{j}\right\|_{\mathcal{C}(\bar{\Omega})}\left\|\psi_{i}\right\|_{H^{1}(\Omega)} .
\end{gathered}
$$

It follows that

$$
\frac{\left\langle L_{i} \psi_{i}, \psi_{i}\right\rangle}{\left\|\psi_{i}\right\|_{H^{1}(\Omega)}} \geq c_{1}\left\|\psi_{i}\right\|_{H^{1}(\Omega)}-c_{2} \tau\left\|w_{j}\right\|_{\mathcal{C}(\bar{\Omega})} \longrightarrow \infty \quad \text { for } \quad\left\|\psi_{i}\right\|_{H^{1}(\Omega)} \rightarrow \infty,
$$

for all $\tau \in[0,1]$, which yields coercivity. Hence, from a theorem by Minty-Browder (cf. [43, Theorem 2.2]), we obtain a unique solution $\psi_{i} \in H^{1}(\Omega)$.

Furthermore, by using Sobolev-Campanato spaces and techniques introduced in $[16,19]$ by Griepentrog, we are able to obtain better regularity for $\psi_{i}$. Due to the Sobolev embeddings we have continuous embeddings $H^{1}(\Omega) \hookrightarrow L^{p}(G), 1 \leq p<\infty$, 
which tells us that $\zeta\left(\psi_{i}, w_{j}\right)$ belongs to $L^{p}(G), 1 \leq p<\infty$, for $G=\Omega, \Gamma$. By reformulating (2.9), we have

$$
\begin{aligned}
& \left(\epsilon^{2} \frac{\mu_{i}^{2}}{\sigma_{t}} \nabla \psi_{i}, \nabla \varphi_{i}\right)_{\Omega}+\tau\left(\beta_{i, i} \psi_{i}, \varphi_{i}\right)_{\Gamma}+\left(\left(\sigma_{t}-\tau \sigma_{s} \omega_{i}\right) \psi_{i}, \varphi_{i}\right)_{\Omega} \\
= & \tau\left(\sigma_{a} I_{b}(\vartheta)+\sigma_{s} \omega_{j} \zeta\left(\psi_{i}, w_{j}\right), \varphi_{i}\right)_{\Omega}+\tau\left(\eta_{i} I_{b}(u)-\beta_{i, j} \zeta\left(\psi_{i}, w_{j}\right), \varphi_{i}\right)_{\Gamma} .
\end{aligned}
$$

Since the right-hand side belongs to the Campanato space of functionals denoted by $Y^{-1,2, \sigma}(\Omega \cup \Gamma)$ with $0<\sigma<2$, we conclude that $\psi_{i}$ belongs to the Sobolev-Campanato space $W_{0}^{1,2, \sigma}(\Omega \cup \Gamma)$ due to [16, Theorem 4.12]. Furthermore, by the embedding theorems for Sobolev-Campanato spaces, the embedding $W_{0}^{1,2, \sigma}(\Omega \cup \Gamma) \hookrightarrow \mathcal{C}^{0, \alpha}(\bar{\Omega})$ is continuous with $\alpha=\sigma / 2$.

Observe that the auxiliary problem induces a compact mapping

$$
\mathrm{H}:[\mathcal{C}(\bar{\Omega})]^{2} \times[0,1] \rightarrow[\mathcal{C}(\bar{\Omega})]^{2} ; \quad(w, \tau) \mapsto \psi,
$$

with $\mathrm{H}(w, 0)=0$ for all $w \in[\mathcal{C}(\bar{\Omega})]^{2}$. Indeed, for given $(w, \tau) \in[\mathcal{C}(\bar{\Omega})]^{2} \times[0,1]$, the system decouples and we are able to solve the equations independently as above. We further obtain $\mathcal{C}^{0, \alpha}(\bar{\Omega})$ regularity for the solutions $\psi_{i}$. Due to well known properties for Hölder spaces, we have that the embedding $\mathcal{C}^{0, \alpha}(\bar{\Omega}) \hookrightarrow \mathcal{C}(\bar{\Omega})$ is compact for $0<\alpha \leq 1$ (cf. [5, Theorem 8.6]).

By a simple Stampacchia argument, we now show uniform boundedness for all $(\psi, \tau) \in[\mathcal{C}(\bar{\Omega})]^{2} \times[0,1]$ satisfying $\psi=\mathrm{H}(\psi, \tau)$. We set $\chi_{i}^{+}=\left\{x \in \bar{\Omega} \mid \psi_{i}(x)>I_{b}(\bar{q})\right\}$. By showing that $\chi_{i}^{+}=\emptyset$, we obtain the upper bound for $\psi_{i}$, i.e., $\psi_{i} \leq I_{b}(\bar{q})$. To do this, we test $(2.9)$ with $\varphi_{i}^{+}=\left(\psi_{i}-I_{b}(\bar{q})\right)^{+}$. This yields the bound

$$
\begin{aligned}
& \epsilon^{2} \frac{\mu_{i}^{2}}{\overline{\sigma_{t}}}\left\|\nabla \varphi_{i}^{+}\right\|_{2, \Omega}^{2}+\tau \eta_{i}\left\|\varphi_{i}^{+}\right\|_{2, \Gamma}^{2}+\left(\underline{\sigma_{a}}+(1-\tau) \underline{\sigma_{s}}\right)\left\|\varphi_{i}^{+}\right\|_{2, \Omega}^{2} \\
\leq & -\tau\left(\sigma_{a}\left(I_{b}(\bar{q})-I_{b}(\vartheta)\right), \varphi^{+}\right)_{\Omega}-\tau \eta_{i}\left(I_{b}(\bar{q})-I_{b}(u), \varphi^{+}\right)_{\Gamma} \leq 0,
\end{aligned}
$$

for all $\tau \in[0,1]$, where we used the fact that $\zeta\left(\psi_{i}, \psi_{j}\right)=\psi_{i}$ for $\psi_{i}>I_{b}(\bar{q})$ and that $\sum_{j} \beta_{i, j}=\eta_{i}$, which shows that $\chi_{i}^{+}=\emptyset$. Similarly, we can show a similar bound for $\chi_{i}^{-}=\left\{x \in \bar{\Omega} \mid \psi_{i}(x)<I_{b}(\underline{q})\right\}$ to conclude that $\chi_{i}^{-}=\emptyset$, i.e., $I_{b}(\underline{q}) \leq \psi_{i}$. Altogether,

$$
I_{b}(\underline{q}) \leq \psi_{i} \leq I_{b}(\bar{q}) \quad \Rightarrow \quad\left\|\psi_{i}\right\|_{\infty, \Omega},\left\|\psi_{i}\right\|_{\infty, \Gamma} \leq M_{\Psi}
$$

with $M_{\Psi}:=\max \left\{\left|I_{b}(\underline{q})\right|,\left|I_{b}(\bar{q})\right|\right\}$, and finally $\left\|\psi_{i}\right\|_{\mathcal{C}(\bar{\Omega})} \leq M_{\Psi}<M_{\Psi}+1$ since the norms coincide for functions in $\mathcal{C}(\bar{\Omega})$.

We now have all the ingredients to utilize the Leray-Schauder fixed point theorem found in [15, Theorem 11.6], which then concludes the existence of fixed points $\psi \in$ $[\mathcal{C}(\bar{\Omega})]^{2}$ for $\tau=1$. Furthermore, these fixed points belong to $\left[H^{1}(\Omega) \cap \mathcal{C}^{0, \alpha}(\bar{\Omega})\right]^{2}$ with $0<\alpha \leq 1$ as given above. We note that $\zeta\left(\psi_{i}, \psi_{j}\right)=\psi_{j}$ for all $\psi_{i}$ with $I_{b}(\underline{q}) \leq \psi_{i} \leq I_{b}(\bar{q})$. Thus these fixed points are solutions to the actual problem, namely (2.8).

Uniqueness. As for the uniqueness, let $\psi^{1}$ and $\psi^{2}$ be solutions to (2.8). Then the difference $\tilde{\psi}=\psi^{1}-\psi^{2}$, due to linearity, solves (2.8) with $F_{\psi} \equiv 0$. Following the arguments above for existence, we have the uniform estimates $0 \leq \tilde{\psi} \leq 0$, which shows that $\psi^{1} \equiv \psi^{2}$ in $[\mathcal{C}(\bar{\Omega})]^{2}$ and hence we obtain uniqueness.

Corollary 2.5. Let Assumption 1 hold, $\vartheta \in L^{\infty}(\Omega)$, and $u \in L^{\infty}(\Gamma)$. Then the solution of (2.6) given by Theorem 2.6 belongs to $\left[H^{1}(\Omega) \cap \mathcal{C}(\bar{\Omega})\right]^{2}$, such that

$$
I_{b}(\underline{q}) \leq \phi \leq I_{b}(\bar{q})
$$


holds true with $\underline{q}$ and $\bar{q}$ as given in Theorem 2.\%.

Proof. This result follows directly from the similarity transformation $\phi=\sum_{i} \omega_{i} \psi_{i}$ and the fact that $\sum_{i} \omega_{i}=1$, which leads to

$$
\underline{q}=\sum_{i} \omega_{i} \underline{q} \leq \sum_{i} \omega_{i} \psi_{i}=\phi=\sum_{i} \omega_{i} \psi_{i} \leq \sum_{i} \omega_{i} \bar{q}=\bar{q},
$$

thus concluding the assertion.

Suppose now that the functions $\vartheta$ and $u$ depend also on time, which is the case for the SNCR system. We then obtain an easy consequence for the solution $\psi$ depending on time, and thus also for the incident radiation $\phi$.

TheOREM 2.8. If Assumption 1 holds and $F_{\Phi}(u, \vartheta) \in\left[V^{*}\right]^{2}$, then the solution $\Phi$ of (2.6) belongs to $[V]^{2}$, such that

$$
\|\Phi\|_{[V]^{2}} \leq c_{\psi}\left\|F_{\Phi}(u, \vartheta)\right\|_{\left[V^{*}\right]^{2}} .
$$

If further $\vartheta \in L^{\infty}(Q)$ and $u \in L^{\infty}(\Sigma)$, then $\phi$ belongs to $L^{\infty}\left(I ; H^{1}(\Omega) \cap \mathcal{C}(\bar{\Omega})\right)$ and satisfies the uniform bounds

$$
I_{b}(\underline{q}) \leq \phi \leq I_{b}(\bar{q})
$$

with $\underline{q}$ and $\bar{q}$ as given in Theorem 2.7.

Proof. This follows from a simple almost everywhere pointwise (in time) argument applied to theorems 2.6 and 2.7.

2.4. Heat equation. Consider the system for temperature $\vartheta$, given by

$$
\begin{aligned}
\partial_{t} \vartheta+v \cdot \nabla \vartheta-\nabla \cdot(\kappa \nabla \vartheta)+\mathrm{Pl}^{-1} \sigma_{a}\left(I_{b}(\vartheta)-\phi\right) & =f_{\vartheta}, & & \text { in } Q, \\
n \cdot \kappa \nabla \vartheta+\gamma \vartheta & =\gamma u, & & \text { on } \Sigma, \\
\vartheta(0) & =\vartheta_{0}, & & \text { in } \Omega,
\end{aligned}
$$

where $v$ is a given velocity field, and $\phi, f_{\vartheta}, u$, and $\vartheta_{0}$ are given functions. We call $\vartheta$ a weak solution to (2.11) if it fulfills the weak formulation, $\vartheta \in \mathcal{W}(V) \cap L^{\infty}(Q)$ :

$$
\left\{\begin{array}{r}
\left\langle\partial_{t} \vartheta+A_{\vartheta}(\vartheta)+B_{\vartheta}(v, \vartheta)+G_{\vartheta}(\vartheta, \phi)-F_{\vartheta}(u), \varphi_{1}\right\rangle=0 \\
\left(\vartheta(0)-\vartheta_{0}, \varphi_{2}\right)_{\Omega}=0
\end{array}\right\} \forall \varphi \in V \times L^{2}(\Omega),
$$

where the operators $A_{\vartheta}, B_{\vartheta}$, and $F_{\vartheta}$ are induced by the forms

$$
\begin{aligned}
\left\langle A_{\vartheta}(\vartheta), \varphi_{1}\right\rangle & =\left(\kappa \nabla \vartheta, \nabla \varphi_{1}\right)_{Q}+\left(\gamma \vartheta, \varphi_{1}\right)_{\Sigma} \\
\left\langle B_{\vartheta}(v, \vartheta), \varphi_{1}\right\rangle & =\left\langle v \cdot \nabla \vartheta, \varphi_{1}\right\rangle \\
\left\langle G_{\vartheta}(\vartheta, \phi), \varphi_{1}\right\rangle & =\left(\mathrm{Pl}^{-1} \sigma_{a}\left(I_{b}(\vartheta)-\phi\right), \varphi_{1}\right)_{Q} \\
\left\langle F_{\vartheta}(u), \varphi_{1}\right\rangle & =\left(f_{\vartheta}, \varphi_{1}\right)_{Q}+\left(\gamma u, \varphi_{1}\right)_{\Sigma}
\end{aligned}
$$

Let us consider the linear, inhomogeneous part of $(2.12)$, namely, $\vartheta_{L} \in \mathcal{W}(V)$ :

$$
\left\{\begin{array}{r}
\left\langle\partial_{t} \vartheta_{L}+A_{\vartheta}\left(\vartheta_{L}\right)+B_{\vartheta}\left(v, \vartheta_{L}\right)-F_{\vartheta}(u), \varphi_{1}\right\rangle=0 \\
\left(\vartheta_{L}(0)-\vartheta_{0}, \varphi_{2}\right)_{\Omega}=0
\end{array}\right\} \forall \varphi \in V \times L^{2}(\Omega),
$$

and state standard results concerning this equation. 
TheOREm 2.9. Given $v \in \mathcal{W}(\mathcal{V}), F_{\vartheta}(u) \in \mathcal{W}(V)^{*}$, and $\vartheta_{0} \in L^{2}(\Omega)$, there exists a unique $\vartheta_{L} \in \mathcal{W}(V)$ fulfilling (2.13).

If moreover $f_{\vartheta} \in L^{\infty}(Q), u \in L^{\infty}(\Sigma)$, and $\vartheta_{0} \in L^{\infty}(\Omega)$, then $\vartheta_{L} \in \mathcal{W}(V) \cap L^{\infty}(Q)$, such that the estimate

$$
\left\|\vartheta_{L}\right\|_{\mathcal{W}(V)}+\left\|\vartheta_{L}\right\|_{\infty, Q} \leq c\left(\left\|f_{\vartheta}\right\|_{\infty, Q}+\|u\|_{\infty, \Sigma}+\left\|\vartheta_{0}\right\|_{\infty, \Omega}\right)
$$

holds for some constant $c>0$ independent of $v, f_{\vartheta}, u$, and $\vartheta_{0}$. In this case, the bounds

$$
\underline{q}_{\vartheta} \leq \vartheta_{L} \leq \bar{q}_{\vartheta}
$$

hold true with $\underline{q}_{\vartheta}=\min \left\{\underline{\vartheta}_{0}, \underline{\gamma} \underline{u}, \underline{f}_{\vartheta}, 0\right\}$ and $\bar{q}_{\vartheta}=e^{T} \max \left\{\bar{\vartheta}_{0}, \bar{\gamma} \bar{u}, \bar{f}_{\vartheta}, 0\right\}$.

Proof. The first assertion follows from standard theory for linear parabolic equations (c.f. $[42,29,43]$ ) and the fact that $v \in\left[L^{4}(Q)\right]^{2}$ due to Theorem 2.2, and thus $B_{\vartheta}(v, \vartheta) \in V^{*}$. This gives us the estimate

$$
\left\|\vartheta_{L}\right\|_{\mathcal{W}(V)} \leq c_{1}\left(\left\|F_{\vartheta}(u)\right\|_{\mathcal{W}(V)^{*}}+\left\|\vartheta_{0}\right\|_{2, \Omega}\right)
$$

where $c_{1}$ is a constant independent of $v, f_{\vartheta}, u$, and $\vartheta_{0}$. The fact that $c_{1}$ is independent of $v$ follows from the fact that $v \in \mathcal{V}$, and hence

$$
(v(t) \cdot \nabla \vartheta(t), \vartheta(t))_{\Omega}=\frac{1}{2}\left(\int_{\Gamma} \vartheta(t)^{2} v(t) \cdot n \mathrm{~d} \mu-\int_{\Omega} \vartheta(t)^{2} \operatorname{div} v(t) \mathrm{d} x\right)=0
$$

for almost every $t \in I$.

The second assertion follows from the maximum principle for parabolic equations and a simple Stampacchia argument. Now let the functions $f_{\vartheta}, u$, and $\vartheta_{0}$ have the above mentioned regularity. We set

$$
\underline{w}=\min \left\{\underline{\vartheta}_{0}, \underline{\gamma} \underline{u}, \underline{f}_{\vartheta}, 0\right\} \quad \text { and } \quad \bar{w}=\max \left\{\bar{\vartheta}_{0}, \bar{h} \bar{\gamma}, \bar{f}_{\vartheta}, 0\right\} .
$$

Define $w:=e^{-t} \vartheta_{L}$. Substituting $\vartheta_{L}=e^{t} w$ into (2.13) and dividing by $e^{t}$, we obtain the problem

$$
w \in \mathcal{W}(V):\left\{\begin{array}{r}
\left\langle\partial_{t} w+A_{\vartheta}(w)+B_{\vartheta}(v, w)-e^{-t} F_{\vartheta}(u), \varphi_{1}\right\rangle=0 \\
\left(w(0)-\vartheta_{0}, \varphi_{2}\right)_{\Omega}=0
\end{array}\right\} \forall \varphi \in V \times L^{2}(\Omega) .
$$

Now consider the set $\chi^{+}=\{(x, t) \in \bar{Q} \mid w(x, t)>\bar{w}\}$ and test $(2.14)$ with $\varphi^{+}=(w-\bar{w})^{+}$. This leads us to the inequality

$$
\frac{1}{2}\left\|\varphi^{+}(t)\right\|_{2, \Omega}^{2}+\int_{0}^{t}\left\|\varphi^{+}\right\|_{2, \Omega}^{2} \mathrm{~d} t \leq-\left(\bar{w}-e^{-t} f_{\vartheta}, \varphi^{+}\right)_{Q}-\left(\bar{w}-e^{-t} \gamma u, \varphi^{+}\right)_{\Sigma} \leq 0
$$

for almost every $t \in I$, and hence $w \leq \bar{w}$. Here we used the fact that

$$
\left\langle A_{\vartheta}\left(\varphi^{+}\right)+B_{\vartheta}\left(v, \varphi^{+}\right), \varphi^{+}\right\rangle \geq 0 .
$$

Similarly, by showing that $\chi^{-}=\{(x, t) \in \bar{Q} \mid w(x, t)<\underline{w}\}$ is empty, we find the bound $\underline{w} \leq w$. Altogether, we have

$$
\underline{w} \leq w \leq \bar{w} \quad \Rightarrow \quad \underline{q}_{\vartheta}=e^{0} \underline{w} \leq \vartheta_{L} \leq e^{T} \bar{w}=\bar{q}_{\vartheta},
$$


and thus $\left\|\vartheta_{L}\right\|_{\infty, Q} \leq M_{\vartheta}:=\max \left\{\left|\underline{q}_{\vartheta}\right|,\left|\bar{q}_{\vartheta}\right|\right\}$, which are the bounds claimed for $\vartheta_{L}$.

Now consider the nonlinear part of (2.12), namely, $\vartheta_{N} \in \mathcal{W}(V) \cap L^{\infty}(Q)$ :

$$
\left\{\begin{aligned}
\left\langle\partial_{t} \vartheta_{N}+A_{\vartheta}\left(\vartheta_{N}\right)+B_{\vartheta}\left(v, \vartheta_{N}\right)+G_{\vartheta}(\vartheta, \phi), \varphi_{1}\right\rangle & =0 \\
\left(\vartheta_{N}(0), \varphi_{2}\right)_{\Omega} & =0
\end{aligned}\right\} \forall \varphi \in V \times L^{2}(\Omega),
$$

Then the solution to (2.12) can be given as the sum of the solutions to (2.13) and (2.15), respectively, i.e., $\vartheta=\vartheta_{L}+\vartheta_{N}$.

2.5. Existence of state vectors. We define the state space and image space as

$$
\begin{aligned}
\mathcal{X}_{\infty} & =\left[\mathcal{W}(\mathcal{V}) \cap\left[L^{\infty}(Q)\right]^{2} \cap \mathcal{C}\left(\bar{I} ; \mathcal{H}^{1}\right)\right] \times\left[\mathcal{W}(V) \cap L^{\infty}(Q)\right] \times\left[L^{\infty}\left(I ; H^{1}(\Omega) \cap \mathcal{C}(\bar{\Omega})\right)\right]^{2}, \\
Z & =\mathcal{V} \times \mathcal{H} \times V \times L^{2}(\Omega) \times[V]^{2},
\end{aligned}
$$

respectively and denote the states by $y=(v, \vartheta, \Phi) \in \mathcal{X}_{\infty}$. Due to the prior discussions, we now have sufficient ingredients to show existence and uniqueness of states in the mentioned space for the full state system, namely, Theorem 2.1.

The state system (1.1) can be written in its weak formulation as

$$
y \in \mathcal{X}_{\infty}: E(u, y)=0 \text { in } Z^{*},
$$

which is explicitly given by

$$
y \in \mathcal{X}_{\infty}:\left\{\begin{array}{r}
\left\langle\partial_{t} v+A_{v}(v)+B_{v}(v)-F_{v}(\vartheta), \varphi_{1}\right\rangle=0 \\
\left(v(0)-v_{0}, \varphi_{2}\right)_{\Omega}=0 \\
\left\langle\partial_{t} \vartheta+A_{\vartheta}(\vartheta)+B_{\vartheta}(v, \vartheta)+G_{\vartheta}(\vartheta, \Phi)-F_{\vartheta}(u), \varphi_{3}\right\rangle=0 \\
\left(\vartheta(0)-\vartheta_{0}, \varphi_{4}\right)_{\Omega}=0 \\
\left\langle A_{\Phi}(\Phi)+B_{\Phi}(\Phi)-F_{\Phi}(u, \vartheta), \varphi_{5}\right\rangle=0
\end{array}\right\} \forall \varphi \in Z .
$$

We further make the following regularity assumptions.

Assumption 2. The data have the following regularity: $f_{v} \in\left[L^{\infty}(Q)\right]^{2}, \sqrt{t} \partial_{t} f_{v} \in \mathcal{V}^{*}$, $v_{0} \in \mathcal{H}^{1} \cap\left[L^{\infty}(\Omega)\right]^{2}, f_{\vartheta} \in L^{\infty}(Q)$, and $\vartheta_{0} \in L^{\infty}(\Omega)$.

Proof of Theorem 2.1. Existence. We wish to apply the Leray-Schauder fixed point theorem stated in [50, Theorem 2.A] to conclude this theorem. For this reason, we will introduce a compact mapping

$$
\mathrm{H}: L^{2}(Q) \times[0,1] \rightarrow L^{2}(Q) ; \quad(w, \tau) \mapsto \vartheta
$$

with $\mathrm{H}(w, 0)=0$ for all $w \in L^{2}(Q)$, which is defined with the help of an auxiliary problem of (2.16). We then show that this mapping is uniformly bounded for all $(w, \tau)$ satisfying $w=\mathrm{H}(w, \tau)$. This is achieved by introducing the cutoff operator $\varrho: L^{2}(Q) \rightarrow$ $L^{2}(Q)$ with $\varrho(w)=\max (\underline{q}, \min (w, \bar{q}))$, where $\underline{q}=\min \left\{\underline{q}_{\vartheta}, \underline{u}, 0\right\}$ and $\bar{q}=\max \left\{\bar{q}_{\vartheta}, \bar{u}, 0\right\}$, with $\underline{q}_{\vartheta}$ and $\bar{q}_{\vartheta}$ are as defined in Theorem 2.9 , and by using maximum principles for each subproblem respectively. Notice that $\varrho(w) \in L^{\infty}(Q)$ and is uniformly bounded for all $w \in L^{2}(Q)$, thereby enabling the use of the maximum principles.

For any $(w, \tau) \in L^{2}(Q) \times[0,1]$, we consider the auxiliary problem given by

$$
y \in \mathcal{X}:\left\{\begin{aligned}
\left\langle\partial_{t} v+A_{v}(v)+B_{v}(v), \varphi_{1}\right\rangle & =\tau\left\langle F_{v}(\varrho(w)), \varphi_{1}\right\rangle \\
\left(v(0), \varphi_{2}\right)_{\Omega} & =\tau\left(v_{0}, \varphi_{2}\right)_{\Omega} \\
\left\langle\partial_{t} \vartheta+A_{\vartheta}(\vartheta)+B_{\vartheta}(v, \vartheta), \varphi_{3}\right\rangle & =\tau\left\langle F_{\vartheta}(u)-G_{\vartheta}(\varrho(w), \Phi), \varphi_{3}\right\rangle \\
\left(\vartheta(0), \varphi_{4}\right)_{\Omega} & =\tau\left(\vartheta_{0}, \varphi_{4}\right)_{\Omega} \\
\left\langle A_{\Phi}(\Phi)+B_{\Phi}(\Phi), \varphi_{5}\right\rangle & =\tau\left\langle F_{\Phi}(u, \varrho(w)), \varphi_{5}\right\rangle
\end{aligned}\right\} \forall \varphi \in Z,
$$


which defines the mapping $\mathrm{H}:(w, \tau) \mapsto \vartheta$. From the auxiliary problem one easily sees that the equations decouple, in the sense that they may be solved simply. Indeed, for any given $w$, the Navier-Stokes equation and the $\mathrm{SP}_{3}$-approximation equations may be solved independently for $v$ and $\Phi$ respectively. More precisely, according to theorems 2.3 and 2.8 , there exist solutions

$$
v \in \mathcal{W}(\mathcal{V}) \cap\left[L^{\infty}(Q)\right]^{2} \quad \text { and } \quad \Phi \in\left[L^{\infty}\left(I ; H^{1}(\Omega) \cap \mathcal{C}(\bar{\Omega})\right)\right]^{2}
$$

to their respective equations. They are then inserted into the heat equation to be solved for $\vartheta$ with right-hand side $F_{\vartheta}(u)-G_{\vartheta}(\varrho(w), \Phi) \in V^{*}$ to obtain $\vartheta \in \mathcal{W}(V)$. Due to a theorem by Lions-Aubin (c.f. [43, Proposition 1.3]), we have the compact embedding $\mathcal{W}(V) \hookrightarrow L^{2}(Q)$, which altogether defines the compact mapping $\mathrm{H}:(w, \tau) \mapsto \vartheta$. The fact that $\mathrm{H}(w, 0)=0$ for all $w \in L^{2}(Q)$ follows directly from theorems 2.2, 2.7, and 2.9.

To show uniform boundedness we let $(\vartheta, \tau) \in L^{2}(Q)$ satisfy $\vartheta=\mathrm{H}(\vartheta, \tau)$. Then

$$
\underline{q} \leq \vartheta \leq \bar{q} \quad \forall \tau \in[0,1] .
$$

Indeed, let $\chi^{+}=\{(x, t) \in \bar{Q} \mid \vartheta(x, t)>\bar{q}\}$. Since we know from Theorem 2.9 that $\vartheta_{L} \leq \bar{q}$, $\vartheta_{N}>\bar{q}-\vartheta_{L} \geq 0$ and we may rewrite $\chi^{+}$as $\chi^{+}=\left\{(x, t) \in \bar{Q} \mid \vartheta_{N}(x, t)>0\right\}$. Using $\varphi_{3}^{+}=$ $\left(\vartheta_{N}\right)^{+}$as a test function for (2.15) and integrating with respect to time leads us to

$$
\left\|\varphi_{3}^{+}(t)\right\|_{2, \Omega}^{2} \leq-2 \tau\left(\mathrm{Pl}^{-1} \sigma_{a}\left(I_{b}(\bar{q})-\phi\right), \varphi_{3}^{+}\right)_{Q} \leq 0
$$

for almost every $t \in I$ and every $\tau \in[0,1]$, and thus $\vartheta_{N} \leq 0$. Here we used the fact that

$$
\left\langle A_{\vartheta}\left(\varphi_{3}^{+}\right)+B_{\vartheta}\left(v, \varphi_{3}^{+}\right), \varphi_{3}^{+}\right\rangle \geq 0
$$

and that $I_{b}(q) \leq \phi \leq I_{b}(\bar{q})$, which is due to Theorem 2.8. Similarly we can show that $\chi^{-}=\left\{(x, t) \in \bar{Q} \mid \vartheta_{N}(x, t)<0\right\}=\emptyset$, which implies $0 \leq \vartheta_{N}$. Altogether we have

$$
\underline{q} \leq \vartheta_{L}+\vartheta_{N}=\vartheta=\vartheta_{L}+\vartheta_{N} \leq \bar{q} \quad \forall \tau \in[0,1],
$$

as required. Furthermore, by setting $M:=\max \{|\underline{q}|,|\bar{q}|\}$, we have

$$
\|\vartheta\|_{2, Q} \leq M<M+1
$$

for all $\vartheta, \tau \in L^{2}(Q) \times[0,1]$ satisfying $\vartheta=\mathrm{H}(\vartheta, \tau)$.

Fulfilling all requirements of the Leray-Schauder fixed point theorem, we obtain the existence of at least one fixed point $\vartheta$ for the mapping $\mathrm{H}(\cdot, 1): L^{2}(Q) \rightarrow L^{2}(Q)$. Since this fixed point $\vartheta$ belongs to $\mathcal{W}(V) \cap L^{\infty}(Q)$ and further fulfills the bounds $\underline{q} \leq \vartheta \leq \bar{q}$, implying that $\varrho(\vartheta)=\vartheta$, it solves the initial problem (2.16).

Uniqueness. Let $y^{1}, y^{2} \in \mathcal{W}(V)$ be two solutions to (2.16). Then the difference $\tilde{y}=y^{1}-y^{2} \in \mathcal{W}(V)$ solves the system, $\tilde{y} \in \mathcal{X}_{\infty}$ :

$$
\left\{\begin{aligned}
\left\langle\partial_{t} \tilde{v}+A_{v}(\tilde{v})+B_{v}\left(\tilde{v}, v^{1}\right)+B_{v}\left(v^{2}, \tilde{v}\right), \varphi_{1}\right\rangle & =\left(\operatorname{RaPr} \tilde{\vartheta}, \varphi_{1}\right)_{Q} \\
\left(\tilde{v}(0), \varphi_{2}\right)_{\Omega} & =0 \\
\left\langle\partial_{t} \tilde{\vartheta}+A_{\vartheta}(\tilde{\vartheta})+B_{\vartheta}\left(\tilde{v}, \vartheta^{1}\right)+B_{\vartheta}\left(v^{2}, \tilde{\vartheta}\right), \varphi_{3}\right\rangle & =\left(\mathrm{Pl}^{-1} \sigma_{a}\left(g\left(\vartheta^{1}, \vartheta^{2}\right)-\tilde{\phi}\right), \varphi_{3}\right)_{Q} \\
\left(\tilde{\vartheta}(0), \varphi_{4}\right)_{\Omega} & =0 \\
\left\langle A_{\Phi}(\tilde{\Phi})+B_{\Phi}(\tilde{\Phi}), \varphi_{5}\right\rangle & =\left(\sigma_{a} g\left(\vartheta^{1}, \vartheta^{2}\right), \varphi_{5}\right)_{Q}
\end{aligned}\right\} \forall \varphi \in Z,
$$

where we have used $g$ to denote $g\left(\vartheta^{1}, \vartheta^{2}\right)=I_{b}\left(\vartheta^{1}\right)-I_{b}\left(\vartheta^{2}\right) \in L^{\infty}(Q)$. 
First of all we observe that, due to Lemma 2.4, there exists a constant $l_{0}(M)>0$ depending on $M$ such that

$$
\left\|g\left(\vartheta^{1}(t), \vartheta^{2}(t)\right)\right\|_{2, \Omega} \leq l_{0}(M)\|\tilde{\vartheta}(t)\|_{2, \Omega} \quad \text { a.e. } t \in I,
$$

where $M:=\max \left\{\left\|\vartheta^{1}\right\|_{\infty, Q},\left\|\vartheta^{2}\right\|_{\infty, Q}\right\}$. From Theorem 2.8, we have $\tilde{\Phi}(t) \in\left[H^{1}(\Omega)\right]^{2}$ and the estimate

$$
\|\tilde{\Phi}(t)\|_{\left[H^{1}(\Omega)\right]^{2}} \leq c_{\Phi} \bar{\sigma}_{a}\left\|g\left(\vartheta^{1}(t), \vartheta^{2}(t)\right)\right\|_{2, \Omega} \leq c_{\Phi} \bar{\sigma}_{a} l_{0}(M)\|\tilde{\vartheta}(t)\|_{2, \Omega} \quad \text { a.e. } t \in I .
$$

Due to Young's inequality and the estimate from Theorem 2.2, we further have

$$
\begin{aligned}
\left(\tilde{v}(t) \cdot \nabla \vartheta^{1}(t), \tilde{\vartheta}(t)\right)_{\Omega} & \leq c_{1}\|\tilde{v}(t)\|_{4, \Omega}\left\|\vartheta^{1}(t)\right\|_{H^{1}(\Omega)}\|\tilde{\vartheta}(t)\|_{H^{1}(\Omega)} \\
& \leq \frac{\delta}{2}\|\tilde{\vartheta}(t)\|_{H^{1}(\Omega)}^{2}+\frac{1}{2 \delta} c_{1}^{2} c_{v}^{2} m^{2}\|\tilde{\vartheta}(t)\|_{2, \Omega}^{2},
\end{aligned}
$$

where we denoted $m=\left\|\vartheta^{1}\right\|_{V}$. Now, by testing the heat equation with $\varphi_{3}(t)=\tilde{\vartheta}(t)$ and using the above inequalities, we obtain

$$
\frac{1}{2} \frac{\mathrm{d}}{\mathrm{d} t}\|\tilde{\vartheta}(t)\|_{2, \Omega}^{2}+\left(c_{\vartheta}-\frac{\delta}{2}\right)\|\tilde{\vartheta}(t)\|_{H^{1}(\Omega)}^{2} \leq\left(\left(1+c_{\Phi} \bar{\sigma}_{a}\right) l_{0}(M)+\frac{1}{2 \delta} c_{1}^{2} c_{v}^{2} m^{2}\right)\|\tilde{\vartheta}(t)\|_{2, \Omega}^{2} .
$$

By choosing $\delta=2 c_{\vartheta}$ and setting $c_{2}=2\left(1+c_{\Phi} \bar{\sigma}_{a}\right) l_{0}(M)+\left(c_{1}^{2} c_{v}^{2} m^{2}\right) / \delta$, we obtain

$$
\frac{\mathrm{d}}{\mathrm{d} t}\|\tilde{\vartheta}(t)\|_{2, \Omega}^{2} \leq c_{2}\|\tilde{\vartheta}(t)\|_{2, \Omega}^{2} \quad \Rightarrow \quad\|\tilde{\vartheta}(t)\|_{2, \Omega} \leq 0 \quad \text { a.e. } t \in I,
$$

due to Gronwall's lemma, which clearly implies $\tilde{\vartheta} \equiv 0$ and hence concludes the theorem. Indeed, if we find that $\tilde{\vartheta} \equiv 0$, then it follows from theorems 2.2 and 2.7 that $\tilde{v} \equiv 0$ and $\tilde{\Phi} \equiv 0$ respectively, i.e., altogether we have $\tilde{y} \equiv 0$, and hence the uniqueness.

REMARK 2.6. In fact we obtain better regularity for $v$, namely, $v \in \mathcal{C}\left(\bar{I} ; \mathcal{H}^{1}\right)$. Indeed, by Assumption 2 and the fact that $\vartheta \in L^{2}(Q)$ and $\partial_{t} \vartheta \in V^{*}$, we have $F_{v}(\vartheta) \in L^{2}(Q)$ and $\sqrt{t} \partial_{t} F_{v}(\vartheta) \in \mathcal{V}^{*}$, which satisfies the requirements of Proposition 2.2.

\section{Existence of an optimal control}

The precise statement of the optimal control problem can be given as follows: Find $\left(u_{*}, y_{*}\right) \in \mathcal{U}_{\text {ad }} \times \mathcal{X}_{\infty}$ :

$$
\left\{\begin{array}{r}
\min J(u, y)=J_{1}(y)+J_{2}(v(T), \vartheta(T))+J_{3}(u) \\
\text { such that }(u, y) \in \mathcal{U}_{\text {ad }} \times \mathcal{X}_{\infty}: E(u, y)=0 \quad \text { in } Z^{*}
\end{array}\right\},
$$

under the following assumptions.

Assumption 3. The set of controls $\mathcal{U}_{a d} \subset W$ is a closed and convex subset of a Banach space $W$, and bounded in the space $L^{\infty}(\Sigma)$. The functionals $J_{1}: \mathcal{X}_{\infty} \rightarrow \mathbb{R}, J_{2}: \mathcal{H} \times$ $L^{2}(\Omega) \rightarrow \mathbb{R}$ and $J_{3}: W \rightarrow \mathbb{R}$ are bounded from below, weakly lower semi-continuous, and Fréchet differentiable with locally Lipschitzian derivative. Further, we require that $J_{3}$ be radially unbounded.

In general a unique minimizer cannot be obtained since the set of solutions for $E(u, y)=0$ in $Z^{*}$ may not be convex. This is indeed the case for our problem due to the nonlinearities involved. Thus, for this minimization problem we only have the following existence result for sufficiently smooth control functions. 
TheOREM 3.1. Under assumptions 1-3 with $W=H^{1}(\Sigma)$, there exists at least one minimizer $\left(u_{*}, y_{*}\right) \in \mathcal{U}_{a d} \times \mathcal{X}_{\infty}$ of the constrained minimization problem (3.1).

Proof. Let $j$ denote the infimum of $J$ in $\mathcal{U}_{\mathrm{ad}} \times \mathcal{X}_{\infty}$. Since $J$ is bounded from below we have a minimizing sequence $\left\{\left(u^{k}, y^{k}\right)\right\}_{k} \subset \mathcal{U}_{\text {ad }} \times \mathcal{X}_{\infty}$ such that

$$
-\infty<j=\liminf _{k \rightarrow \infty} J\left(u^{k}, y^{k}\right) \text { and } E\left(u^{k}, y^{k}\right)=0 \text { in } Z^{*} .
$$

Since $\mathcal{U}_{\text {ad }}$ is closed and convex as a subset of a reflexive space $H^{1}(\Sigma)$ and bounded as a subset of $L^{\infty}(\Sigma)$, there exists a subsequence $\left\{u^{k}\right\}_{k} \subset \mathcal{U}_{\text {ad }}$ and $u_{*} \in \mathcal{U}_{\text {ad }}$ such that

$$
u^{k} \rightarrow u_{*} \text { in } \mathcal{U}_{\mathrm{ad}} \text { and } u^{k} \rightarrow^{*} u_{*} \text { in } L^{\infty}(\Sigma) .
$$

Due to the compact embedding $H^{1}(\Sigma) \hookrightarrow L^{2}(\Sigma)$, and hence the strong convergence in $L^{2}(\Sigma)$, we obtain a subsequence with $u^{k}(x) \rightarrow u_{*}(x)$ for a.e. $x \in \Sigma$.

From (4.11) we also find that $\left\{y^{k}\right\}_{k} \subset \mathcal{X}_{\infty}$ is bounded. Due to the reflexivity of the spaces $\mathcal{W}(\mathcal{V}), \mathcal{W}(V)$, and $L^{2}\left(I ; H^{1}(\Omega)\right)$ we find a subsequence $\left\{y^{k}\right\}_{k} \subset \mathcal{X}_{\infty}$ and an element $y_{*} \in \mathcal{X}_{\infty}$ such that $y^{k} \rightarrow y_{*}$ in $\mathcal{X}_{\infty}$, i.e.,

$$
\begin{aligned}
y^{k} \rightarrow y_{*} \text { in } \mathcal{V} \times V \times[V]^{2}, & y^{k} \rightarrow^{*} y_{*} \text { in }\left[L^{\infty}(Q)\right]^{5}, \\
\partial_{t} v^{k} \rightarrow \partial_{t} v_{*} \text { in } \mathcal{V}^{*}, & \partial_{t} \vartheta^{k}-\partial_{t} \vartheta_{*} \text { in } V^{*}, \\
v^{k}(0)-v_{*}(0) \text { in } \mathcal{H}, & \vartheta^{k}(0)-\vartheta_{*}(0) \text { in } L^{2}(\Omega), \\
v^{k}(T) \rightarrow v_{*}(T) \text { in } \mathcal{H}, & \vartheta^{k}(T)-\vartheta_{*}(T) \text { in } L^{2}(\Omega), \\
v^{k} \rightarrow v_{*} \text { in } L^{2}(I ; \mathcal{H}), & \vartheta^{k} \rightarrow \vartheta_{*} \text { in } L^{2}(Q),
\end{aligned}
$$

where the last two convergences follow from compact embeddings $\mathcal{W}(\mathcal{V}) \hookrightarrow L^{2}(I ; \mathcal{H})$ and $\mathcal{W}(V) \hookrightarrow L^{2}(Q)$ respectively, due to a theorem by Lions-Aubin (cf. [43, Proposition 1.3]). Note that the weak convergences for $\left\{v^{k}(0)\right\}_{k},\left\{\vartheta^{k}(0)\right\}_{k},\left\{v^{k}(T)\right\}_{k}$, and $\left\{\vartheta^{k}(T)\right\}_{k}$ follow from the embeddings $\mathcal{W}(\mathcal{V}) \hookrightarrow \mathcal{C}(\bar{I} ; \mathcal{H})$ and $\mathcal{W}(V) \hookrightarrow \mathcal{C}\left(\bar{I} ; L^{2}(\Omega)\right)$ respectively. As for $\left\{\Phi^{k}\right\}_{k}$ we simply have the weak convergence of a subsequence $\Phi^{k} \rightarrow \Phi_{*}$ in $L^{2}\left(I ; H^{1}(\Omega)\right)$.

Due to the weak convergences obtained above, we easily obtain the weak convergence $B_{v}\left(v^{k}\right) \rightarrow B_{v}\left(v_{*}\right)$ in $\mathcal{V}^{*}$. Indeed, is $\varphi_{1} \in \mathcal{V}$ is arbitrary, then

$$
\begin{aligned}
& \left|\left\langle v^{k} \cdot \nabla v_{j}^{k}-v_{*} \cdot \nabla v_{*, j}, \varphi_{1, j}\right\rangle\right| \\
= & \left|\left\langle v^{k} v_{j}^{k}-v_{*} v_{*, j}, \nabla \varphi_{1, j}\right\rangle\right| \\
= & \left|\left\langle v^{k}\left(v_{j}^{k}-v_{*, j}\right)+\left(v^{k}-v_{*}\right) v_{*, j}, \nabla \varphi_{1, j}\right\rangle\right| \\
\leq & \left(\left\|v^{k}\right\|_{\infty, Q}\left\|v_{j}^{k}-v_{*, j}\right\|_{2, Q}+\left\|v_{*, j}\right\|_{\infty, Q}\left\|v^{k}-v_{*}\right\|_{2, Q}\right)\left\|\nabla \varphi_{1, j}\right\|_{2, Q} \\
& \longrightarrow 0 \quad \text { for } k \longrightarrow \infty, \quad j \in\{1,2\},
\end{aligned}
$$

due to the strong convergence for $v^{k} \rightarrow v_{*}$ in $L^{2}(Q)$ and the boundedness of $\left\{v^{k}\right\}_{k} \subset$ $\left[L^{\infty}(Q)\right]^{2}$. One can easily show the weak convergence $B_{\vartheta}\left(v^{k}, \vartheta^{k}\right) \rightarrow B_{\vartheta}\left(v_{*}, \vartheta_{*}\right)$ in $V^{*}$ in a similar manner. As for the other nonlinear terms, we have the weak convergences

$$
G_{\vartheta}\left(\vartheta^{k}, \Phi^{k}\right) \rightarrow G_{\vartheta}\left(\vartheta_{*}, \Phi_{*}\right) \text { in } V^{*} \text { and } F_{\Phi}\left(u^{k}, \vartheta^{k}\right) \rightarrow F_{\Phi}\left(u_{*}, \vartheta_{*}\right) \text { in } V^{*},
$$

due to Lemma 2.5. Gathering all the results above allows us to pass to the limit in $k$ to obtain the weak convergence $E\left(u^{k}, y^{k}\right) \rightarrow E\left(u_{*}, y_{*}\right)$ in $Z^{*}$, and thus

$$
\left\|E\left(u_{*}, y_{*}\right)\right\|_{Z^{*}} \leq \liminf _{k \rightarrow \infty}\left\|E\left(u^{k}, y^{k}\right)\right\|_{Z^{*}}=0 \quad \Longrightarrow \quad E\left(u_{*}, y_{*}\right)=0 \text { in } Z^{*} .
$$


Finally, from the weak lower semi-continuity of $J$, we have

$$
J\left(u_{*}, y_{*}\right) \leq \liminf _{k \rightarrow \infty} J\left(u^{k}, y^{k}\right)=j \quad \Longrightarrow \quad J\left(u_{*}, y_{*}\right)=j,
$$

which completes the proof.

Remark 3.1. Clearly, the cost functional introduced in (1.3) satisfies Assumption 3. To see that (1.4) is bounded from below, it is sufficient to know that the control-tostate map is bounded. This is indeed the case (c.f. Theorem 2.1, Section 4.2). Hence, (1.4) also satisfies Assumption 3. Thus the existence of minimizers for each of the functionals is guaranteed by Theorem 3.1.

\section{First-order optimality condition}

In this section we derive the first-order optimality condition for a minimizer of the problem (3.1). The main task for the derivation of the optimality system is to show the existence of Lagrange multipliers. To ensure this, we provide a thorough analysis of the linearized state system.

Suppose the requirements of Theorem 2.1 are satisfied. Then for any $u \in \mathcal{U}_{\mathrm{ad}}$, we obtain a unique state $y \in \mathcal{X}_{\infty}$, such that $E(u, y)=0$ in $Z^{*}$. This implicitly defines a control-to-state map $S: u \mapsto y$, which allows us to introduce the reduced cost functional and deduce the first-order optimality condition for the reduced optimal control problem, which reads as follows:

$$
\min \hat{J}(u) \text { subject to } u \in \mathcal{U}_{\text {ad }},
$$

where $\hat{J}(u)=J(u, S(u)): \mathcal{U}_{\mathrm{ad}} \rightarrow \mathbb{R}$. For the reduced optimal control problem (4.1) we have the following characterization of minimizers.

Proposition 4.1. Let $\hat{J}: \mathcal{U}_{a d} \rightarrow \mathbb{R}$ be Gâteaux differentiable in an open neighborhood of the closed and convex set $\mathcal{U}_{a d}$ and $u_{*} \in \mathcal{U}_{a d}$ be a solution of the reduced optimal control problem (4.1). Then

$$
\left\langle\mathrm{d} \hat{J}\left(u_{*}\right), u-u_{*}\right\rangle_{W} \geq 0 \quad \forall u \in \mathcal{U}_{a d} .
$$

Let us assume for a moment that the control-to-state map $S$ is Frechét differentiable. Then $\hat{J}$ is Frechét differentiable and its derivative is given by

$$
\mathrm{d} \hat{J}(u)\left[w_{u}\right]=\left\langle\mathrm{d}_{y} J(u, S(u)), \mathrm{d} S(u)\left[w_{u}\right]\right\rangle_{\mathcal{X}}+\left\langle\mathrm{d}_{u} J(u, S(u)), w_{u}\right\rangle_{W} \quad \forall w_{u} \in W,
$$

where the derivative $\mathrm{d} S$ is given by

$$
\mathrm{d} S(u)\left[w_{u}\right]=-\mathrm{d}_{y} E(u, S(u))^{-1}\left[\mathrm{~d}_{u} E(u, S(u))\right]\left[w_{u}\right] \quad \forall w_{u} \in W .
$$

Furthermore, if there exists a unique adjoint state $z \in Z$ to the adjoint problem

$$
\mathrm{d}_{y} E(u, S(u))^{*}[z]=-\mathrm{d}_{y} J(u, S(u)) \text { in } Z^{*},
$$

then the derivative $\mathrm{d} \hat{J}$ may then by written as

$$
\mathrm{d} \hat{J}(u)\left[w_{u}\right]=\left\langle\mathrm{d}_{u} E(u, y)^{*}[z]+\mathrm{d}_{u} J(u, y), w_{u}\right\rangle_{W} \quad \forall w_{u} \in W .
$$

Summarizing the discussion above, along with Proposition 4.1, we obtain the following characterization of local minimizers of the problem (3.1). 
THEOREM 4.1. Let $\left(u_{*}, y_{*}\right) \in \mathcal{U}_{a d} \times \mathcal{X}_{\infty}$ be a solution of the constrained minimization problem (3.1). Then, there exists a unique Lagrange multiplier $z_{*} \in Z$, which together with the optimal solution $\left(u_{*}, y_{*}\right)$ satisfies the first-order optimality system

$$
\begin{aligned}
E\left(u_{*}, y_{*}\right) & =0, & & \text { in } Z^{*}, \\
\mathrm{~d}_{y} E\left(u_{*}, y_{*}\right)^{*}\left[z_{*}\right]+\mathrm{d}_{y} J\left(u_{*}, y_{*}\right) & =0, & & \text { in } \mathcal{X}^{*}, \\
\left\langle\mathrm{~d}_{u} E\left(u_{*}, y_{*}\right)^{*}\left[z_{*}\right]+\mathrm{d}_{u} J\left(u_{*}, y_{*}\right), u-u_{*}\right\rangle & \geq 0, & & \forall u \in \mathcal{U}_{a d} .
\end{aligned}
$$

The remaining subsections in this section are dedicated to resolving the assumptions needed to prove Theorem 4.1, namely, the continuous Frechét differentiability of the control-to-state map $S$ and the unique solvability of the adjoint problem.

EXAMPLE 4.2. Consider the case where the set of admissible controls is given by

$$
\mathcal{U}_{a d}=\left\{u \in W \mid u_{a} \leq u \leq u_{b}\right\}, \quad u_{a}, u_{b} \in W,
$$

with $u_{a} \leq u_{b}$ a.e. in $\Sigma$ and $W=L^{2}(\Sigma)$ or $W=H^{1}(\Sigma)$. Clearly $\mathcal{U}_{a d}$ satisfies Assumption 3. Now let $\nabla \hat{J}(u) \in W$ denote the Riesz-representation of $\mathrm{d} \hat{J}(u) \in W^{*}$ and $\mathcal{P}_{\mathcal{U}_{a d}}$ denote the projection onto $\mathcal{U}_{a d}$. Then the first-order optimality condition (4.2) may be expressed in the more convenient form

$$
\forall \varrho>0: u_{*}=\mathcal{P}_{\mathcal{u}_{a d}}\left(u_{*}-\varrho \nabla \hat{J}\left(u_{*}\right)\right) .
$$

The proof for this equivalence may be found, e.g., in [25, Lemma 1.11]. In the case $W=L^{2}(\Sigma)$, the projection $\mathcal{P}_{\mathcal{U}_{a d}}(u)=\max \left\{u_{a}, \min \left\{u, u_{b}\right\}\right\}$.

4.1. Linear state vectors. Here, we deal with the linearized state system. We begin by showing that this system is weakly solvable, and further show that the solutions are uniformly bounded for appropriately prescribed data. First of all, we observe from Remark 2.1 and Lemma 2.4 that the state system is continuously Frechét differentiable as an operator from $\mathcal{X}_{\infty}$ to the dual space $Z^{*}$. Furthermore, we know that $\mathcal{X}_{\infty} \hookrightarrow \mathcal{X}$ is a dense embedding, where

$$
\mathcal{X}=\mathcal{W}(\mathcal{V}) \times \mathcal{W}(V) \times[V]^{2} .
$$

So from the BLT theorem (cf. [42, Theorem 8.7]) we have for each $(u, y) \in \mathcal{U}_{\text {ad }} \times \mathcal{X}_{\infty}$ an extension of the operator $\mathrm{d}_{y} E(u, y) \in \mathcal{L}\left(\mathcal{X}_{\infty} ; Z^{*}\right)$, denoted by $L_{u, y} \in \mathcal{L}\left(\mathcal{X} ; Z^{*}\right)$.

By denoting $w_{y}=\left(w_{v}, w_{\vartheta}, w_{\Phi}\right) \in \mathcal{X}$ and $h=\left(h_{v}, w_{v, 0}, h_{\vartheta}, w_{\vartheta, 0}, h_{\Phi}\right) \in Z^{*}$, the weak formulation of the linearized state system may then be written as

$$
w_{y} \in \mathcal{X}: \quad L_{u, y}[w]=h \quad \text { in } Z^{*},
$$

which is explicitly given by

$$
w_{y} \in \mathcal{X}:\left\{\begin{aligned}
\left\langle\partial_{t} w_{v}+A_{v}\left[w_{v}\right]+\mathrm{d} B_{v}(y)\left[w_{y}\right]-\mathrm{d} F_{v}(y)\left[w_{y}\right], \varphi_{1}\right\rangle & =\left\langle h_{v}, \varphi_{1}\right\rangle \\
\left(w_{v}(0), \varphi_{2}\right)_{\Omega} & =\left(w_{v, 0}, \varphi_{2}\right)_{\Omega} \\
\left\langle\partial_{t} w_{\vartheta}+A_{\vartheta}\left[w_{\vartheta}\right]+\mathrm{d} B_{\vartheta}(y)\left[w_{y}\right]+\mathrm{d} G_{\vartheta}(y)\left[w_{y}\right], \varphi_{3}\right\rangle & =\left\langle h_{\vartheta}, \varphi_{3}\right\rangle \\
\left(w_{\vartheta}(0), \varphi_{4}\right)_{\Omega} & =\left(w_{\vartheta, 0}, \varphi_{4}\right)_{\Omega} \\
\left\langle A_{\Phi}\left[w_{\Phi}\right]+B_{\Phi}\left[w_{\Phi}\right]-\mathrm{d}_{y} F_{\Phi}(u, y)\left[w_{y}\right], \varphi_{5}\right\rangle & =\left\langle h_{\Phi}, \varphi_{5}\right\rangle
\end{aligned}\right\} \forall \varphi \in Z
$$

where the operators $\mathrm{d} B_{v}(y), \mathrm{d} F_{v}(y), \mathrm{d} B_{\vartheta}(y), \mathrm{d} G_{\vartheta}(y)$, and $\mathrm{d}_{\vartheta} F_{\Phi}(u, y)$ for all $y \in \mathcal{X}_{\infty}$ are given by

$$
\left\langle\mathrm{d} B_{v}(y)\left[w_{y}\right], \varphi_{1}\right\rangle=\left\langle w_{v} \cdot \nabla v+v \cdot \nabla w_{v}, \varphi_{1}\right\rangle
$$




$$
\begin{aligned}
\left\langle\mathrm{d} F_{v}(y)\left[w_{y}\right], \varphi_{1}\right\rangle & =\left(\operatorname{RaPr} w_{\vartheta} e_{2}, \varphi_{1}\right)_{Q}, \\
\left\langle\mathrm{~d} B_{\vartheta}(y)\left[w_{y}\right], \varphi_{3}\right\rangle & =\left\langle w_{v} \cdot \nabla \vartheta+v \cdot \nabla w_{\vartheta}, \varphi_{3}\right\rangle, \\
\left\langle\mathrm{d} G_{\vartheta}(y)\left[w_{y}\right], \varphi_{3}\right\rangle & =\left(\mathrm{Pl}^{-1} \sigma_{a}\left(\mathrm{~d} I_{b}(\vartheta)\left[w_{\vartheta}\right]-w_{\Phi, 1}\right), \varphi_{3}\right)_{Q}, \\
\left\langle\mathrm{~d}_{y} F_{\Phi}(u, y)\left[w_{y}\right], \varphi_{5}\right\rangle & =\left(\sigma_{a} \mathrm{~d} I_{b}(\vartheta)\left[w_{\vartheta}\right] e_{1}, \varphi_{5}\right)_{Q} .
\end{aligned}
$$

THEOREM 4.2. Let assumptions 1-2 hold and $(u, y) \in \mathcal{U}_{a d} \times \mathcal{X}_{\infty}$. Then the linear operator $L_{u, y} \in \mathcal{L}\left(\mathcal{X} ; Z^{*}\right)$ is a homeomorphism, i.e., for any given $h \in Z^{*}$ there exists a unique $w_{y} \in \mathcal{X}$ solving (4.7).

Proof. By letting $w_{\vartheta} \in L^{2}(Q)$, we see that the linearized Navier-Stokes equation and $\mathrm{SP}_{3}$-approximation equations are uniquely solvable. Indeed, by testing the linearized Navier-Stokes equation with $\varphi_{1}=w_{v}$, we see that the inequality

$$
\partial_{t}\left\|w_{v}(t)\right\|_{\mathcal{H}}^{2}+\lambda_{v, 1}\left\|w_{v}(t)\right\|_{\mathcal{H}^{1}}^{2}-\lambda_{v, 2}\left\|w_{v}(t)\right\|_{\mathcal{H}}^{2} \leq \frac{1}{\delta_{v}}\left(\operatorname{Ra}^{2} \operatorname{Pr}^{2}\left\|w_{\vartheta}(t)\right\|_{2, \Omega}^{2}+\left\|h_{v}(t)\right\|_{\mathcal{H}^{-1}}^{2}\right)
$$

holds a.e. $t \in I$ with $\delta_{v}<\operatorname{Pr} \underline{\nu}$, where

$$
\lambda_{v, 1}=2\left(\operatorname{Pr} \underline{\nu}-\delta_{v}\right) \quad \text { and } \quad \lambda_{v, 2}=\left(2\|v\|_{\mathcal{V}}^{2} / \delta_{v}+\delta_{v}\right),
$$

which is an inequality of Gårding type (cf. [42, Theorem 9.17]). This yields the unique solution $w_{v} \in \mathcal{W}(\mathcal{V})$ by [42, Theorem 11.3] with initial condition $w_{v}(0)=w_{v, 0} \in \mathcal{H}$.

As for the $\mathrm{SP}_{3}$-approximation equations, we directly get a unique solution $w_{\Phi} \in V$, due to Theorem 2.8, along with the estimate

$$
\left\|w_{\Phi}\right\|_{V} \leq c_{\Phi}\left(\bar{\sigma}_{a}\left\|d I_{b}(\vartheta)\right\|_{\infty, Q}\left\|w_{\vartheta}\right\|_{2, Q}+\left\|h_{\Phi}\right\|_{V^{*}}\right) .
$$

Finally, by testing the linearized heat equation with $\varphi_{3}=w_{\vartheta}$ and using the inequalities given above, we obtain the existence of $M=M\left(\|y\|_{\mathcal{X}},\|h\|_{Z^{*}}\right)>0$ and constants $\lambda_{\vartheta, 1}, \lambda_{\vartheta, 2}>0$ such that

$$
\partial_{t}\left\|w_{\vartheta}(t)\right\|_{2, \Omega}^{2}+\lambda_{\vartheta, 1}\left\|w_{\vartheta}(t)\right\|_{\mathcal{H}^{1}}^{2}-\lambda_{\vartheta, 2}\left\|w_{\vartheta}(t)\right\|_{2, \Omega}^{2} \leq M \quad \text { a.e. } t \in I,
$$

and thus the existence of a unique solution to the linearized heat equation with initial condition $w_{v}(0)=w_{v, 0} \in L^{2}(\Omega)$, and hence to (4.7) by [42, Theorem 11.3].

4.1.1. Regularity of linear state vectors. The previous theorem provides only weak regularity of the linear state vector, which can only be increased by assuming more regularity of the right hand side. The regularity of the linear state vectors will be essential to show differentiability properties for the control-to-state map.

TheOREM 4.3. Let assumptions 1-2 hold and $(u, y) \in \mathcal{U}_{a d} \times \mathcal{X}_{\infty}$. The linear operator $L_{u, y} \in \mathcal{L}\left(\mathcal{X}_{\infty} ; \mathcal{Y}\right)$ is a homeomorphism, where

$$
\mathcal{Y}:=\left[L_{2}^{\sigma}(Q)\right]^{2} \times\left[\mathcal{H}^{1} \cap\left[L^{\infty}(\Omega)\right]^{2}\right] \times L_{2}^{\sigma}(Q) \times L^{\infty}(\Omega) \times\left[L^{\infty}(Q)\right]^{2}
$$

for some $\sigma>0$.

Remark 4.3. Recall that $L_{2}^{\sigma}(Q)$ is the Morrey space (c.f. [26]) defined by

$$
L_{2}^{\sigma}(Q)=\left\{\left.\varphi \in L^{2}(Q)\left|[\varphi]_{2, \sigma, Q}^{2}:=\sup _{a \in Q, r>0} \frac{1}{r^{\sigma}} \iint_{Q \cap B_{r}(a)}\right| \varphi\right|^{2} \mathrm{~d} x \mathrm{~d} t<\infty\right\},
$$


and equipped with the norm

$$
\|\varphi\|_{2, \sigma, Q}^{2}=\|\varphi\|_{2, Q}^{2}+[\varphi]_{2, \sigma, Q}^{2} .
$$

Proof. Since $v$ and $w_{v}$ belong to $\mathcal{V}$, we may rewrite $\mathrm{d} B_{v}[y]$ and $\mathrm{d} B_{\vartheta}[y]$ as follows:

$$
\begin{aligned}
& \left\langle\mathrm{d} B_{v}(y)[w], \varphi_{1}\right\rangle=-\left\langle w_{v} \otimes v+v \otimes w_{v}, \nabla \varphi_{1}\right\rangle=-\sum_{i}\left\langle w_{v} v_{i}+v w_{v, i}, \nabla \varphi_{1, i}\right\rangle, \\
& \left\langle\mathrm{d} B_{\vartheta}(y)[w], \varphi_{3}\right\rangle=-\left\langle w_{v} \otimes \vartheta+v \otimes w_{\vartheta}, \nabla \varphi_{3}\right\rangle=-\left\langle w_{v} \vartheta+v w_{\vartheta}, \nabla \varphi_{3}\right\rangle,
\end{aligned}
$$

where $\otimes$ is the canonical tensor product. We now write the linearized Navier-Stokes system as

$$
\begin{aligned}
\left\langle\partial_{t} w_{v, i}+A_{v, i}\left[w_{v, i}\right], \varphi_{1, i}\right\rangle= & \left\langle w_{v} v_{i}+v w_{v, i}, \nabla \varphi_{1, i}\right\rangle \\
& +\left(\operatorname{RaPr} w_{\vartheta} \delta_{2, i}+h_{v, i}, \varphi_{1, i}\right)_{Q}, \\
\left(w_{v, i}(0), \varphi_{2, i}\right)_{\Omega}= & \left(w_{v, 0, i}, \varphi_{2, i}\right)_{\Omega}
\end{aligned}
$$

for $i \in\{1,2\}$ with Kronecker delta $\delta_{i, j}$. For the first term on the right-hand side of (4.8), we obtain by Hölder's inequality and Proposition 2.2 the estimates

$$
\left\|w_{v} v_{i}\right\|_{4, Q} \leq\left\|w_{v}\right\|_{4, Q}\left\|v_{i}\right\|_{\infty, Q} \quad \text { and } \quad\left\|v w_{v, i}\right\|_{4, Q} \leq\|v\|_{\infty, Q}\left\|w_{v, i}\right\|_{4, Q} .
$$

Due to embedding theorems for Lebesgue spaces into Morrey spaces (c.f. [26]),

$$
w_{v} v_{i}+v w_{v, i} \in L_{2}^{\sigma_{1}}(Q) \text { and } \operatorname{RaPr} w_{\vartheta} \delta_{2, i}+h_{v, i} \in L_{2}^{\sigma_{1}-2}(Q),
$$

for some $\sigma_{1} \in(0,2)$ and thus the right-hand side, which we denote by $\tilde{h}_{v}$, is in $\left[L_{2}^{\sigma_{1}}\left(I ; H^{-1}(\Omega)\right)\right]^{2}$. Consider now the problem

$$
\begin{aligned}
\left\langle\partial_{t} w_{v}^{0}+A_{v}\left[w_{v}^{0}\right], \varphi_{1}\right\rangle & =0 \\
\left(w_{v}^{0}(0), \varphi_{2}\right)_{\Omega} & =\left(w_{v, 0}, \varphi_{2}\right)_{\Omega} .
\end{aligned}
$$

Since $w_{v, 0} \in \mathcal{H}^{1} \cap\left[L^{\infty}(\Omega)\right]^{2}$ and $A_{v}$ satisfies the requirements of [45, Theorem 3.3], we obtain a unique $w_{v}^{0} \in \mathcal{W}(\mathcal{V}) \cap\left[L^{\infty}(Q)\right]^{2} \cap \mathcal{C}\left(\bar{I} ; \mathcal{H}^{1}\right) \hookrightarrow\left[L_{2}^{\sigma_{2}}(Q)\right]^{2}$ for some $\sigma_{2}>2$.

By writing $w_{v}=w_{v}^{0}+w_{v}^{1}$ and taking the difference of (4.8) and (4.9), we have

$$
\begin{aligned}
\left\langle\partial_{t} w_{v, i}^{1}+A_{v, i}\left[w_{v, i}^{1}\right], \varphi_{1, i}\right\rangle & =\left\langle\tilde{h}_{v, i}, \varphi_{1, i}\right\rangle, \\
\left(w_{v, i}^{1}(0), \varphi_{2, i}\right)_{\Omega} & =0 .
\end{aligned}
$$

From the theory of maximal regularity in Sobolev-Morrey spaces and the fact that the right-hand side $\tilde{h}_{v} \in\left[L_{2}^{\sigma_{1}}\left(I ; H^{-1}(\Omega)\right)\right]^{2}$, we find that $w_{v}^{1} \in\left[L_{2}^{\sigma_{2}}(Q)\right]^{2}$ for some $\sigma_{2}>2$ due to [17, Theorem 12.1] and [18, Theorem 6.8]. Altogether, we have better regularity for $w_{v}$, i.e., $w_{v}=w_{v}^{0}+w_{v}^{1} \in\left[L_{2}^{\sigma_{2}}(Q)\right]^{2}$.

Iterating this procedure, we find that $\tilde{h}_{v} \in\left[L_{2}^{\sigma_{2}}\left(I ; H^{-1}(\Omega)\right)\right]^{2}$, since

$$
w_{v} v_{i}+v w_{v, i} \in L_{2}^{\sigma_{2}}(Q) \text { and } \operatorname{RaPr} w_{\vartheta} \delta_{2, i}+h_{v, i} \in L_{2}^{\sigma_{2}-2}(Q) .
$$

This leads us to even better regularity for $w_{v}^{1}$, namely

$$
w_{v, i}^{1} \in L_{2}^{\sigma_{2}+2}(Q) \hookrightarrow \mathcal{C}\left(\bar{I} ; \mathcal{C}^{0, \alpha}(\bar{\Omega})\right) \cap \mathcal{C}^{0, \alpha / 2}(\bar{I} ; \mathcal{C}(\bar{\Omega})) \hookrightarrow L^{\infty}(Q),
$$


with $\alpha=\left(\sigma_{2}-4\right) / 2$ due to [17, Theorem 13.5] and [18, Theorem 3.6], and hence $w_{v} \in$ $\left[L^{\infty}(Q)\right]^{2}$. From this result, we further have that $w_{v} \in \mathcal{C}\left(\bar{I} ; \mathcal{H}^{1}\right)$. Indeed, since

$$
-w_{v} \cdot \nabla v-v \cdot \nabla w_{v}+\operatorname{RaPr} w_{\vartheta} e_{2}+h_{v} \in\left[L^{2}(Q)\right]^{2} \quad \text { and } \quad w_{v, 0} \in \mathcal{H}^{1},
$$

we have the required regularity due to [45, Theorem 3.3]. Altogether, $w_{v} \in \mathcal{W}(\mathcal{V}) \cap$ $\left[L^{\infty}(Q)\right]^{2} \cap \mathcal{C}\left(\bar{I} ; \mathcal{H}^{1}\right)$. Following the arguments made above, one obtains the required regularity for the solution to the heat equation, namely $w_{\vartheta} \in \mathcal{W}(V) \cap L^{\infty}(Q)$ since

$$
\tilde{h}_{\vartheta}=\mathrm{d} B_{\vartheta}(y)\left[w_{y}\right]+\mathrm{d} G_{\vartheta}(y)\left[w_{y}\right]+h_{\vartheta} \in L_{2}^{\sigma_{2}}(Q) \quad \text { and } \quad w_{\vartheta, 0} \in L^{\infty}(\Omega),
$$

with $\sigma_{2}>2$ given above. Due to Theorem 2.8, $w_{\Phi} \in\left[L_{\infty}\left(I ; H^{1}(\Omega) \cap \mathcal{C}(\bar{\Omega})\right)\right]^{2}$ since

$$
\tilde{h}_{\Phi}=\mathrm{d}_{y} F_{\Phi}(u, y)\left[w_{y}\right]+h_{\Phi} \in\left[L^{\infty}(Q)\right]^{2} .
$$

Summarizing the results above yields $w_{y} \in \mathcal{X}_{\infty}$, which was to be shown.

4.2. Differentiability of control-to-state map. Recall the results shown at the end of Section 2, which states that for a given $u \in \mathcal{U}_{\mathrm{ad}} \subset L^{\infty}(\Sigma)$, we obtain a solution $y \in \mathcal{X}_{\infty}$ to the state system (2.16). In fact, Theorem 2.1 introduces a controlto-state map given by

$$
S: \mathcal{U}_{\mathrm{ad}} \longrightarrow \mathcal{X}_{\infty} ; \quad u \mapsto y \quad \text { with } \quad\|y\|_{\mathcal{X}_{\infty}} \leq M\left(\|u\|_{\mathcal{U}_{\mathrm{ad}}}\right),
$$

where $M: \mathbb{R}^{+} \rightarrow \mathbb{R}$ is a non-decreasing, locally bounded, monotone function, deduced from theorems $2.2,2.3,2.8$, and 2.1. In this subsection, we will further show that this map is Fréchet differentiable, i.e., we will prove the following theorem.

TheOREM 4.4. Let assumptions 1-2 hold. The control-to-state map given in (4.11) is Fréchet differentiable with $\mathrm{d} S: \mathcal{U}_{a d} \rightarrow \mathcal{L}\left(\mathcal{U}_{a d} ; \mathcal{X}_{\infty}\right)$, and its derivative can be given by

$$
\mathrm{d} S(u)\left[w_{u}\right]=-\mathrm{d}_{y} E(u, y)^{-1}\left[\mathrm{~d}_{u} E(u, y)\right]\left[w_{u}\right] \quad \forall w_{u} \in \mathcal{U}_{a d},
$$

at $(u, y) \in \mathcal{U}_{a d} \times \mathcal{X}_{\infty}$.

Proof. We adopt the ideas introduced in the papers [33, 38]. Consider the state system (2.16). We may split the operators into two parts, such that

$$
(u, y) \in \mathcal{U}_{\mathrm{ad}} \times \mathcal{X}_{\infty}: \quad E(u, y)=K[y]+N(u, y)=0 \text { in } Z^{*},
$$

where the operator $K: \mathcal{X} \rightarrow Z^{*}$ is given by

$$
\langle K(y), \varphi\rangle=\left\{\begin{array}{r}
\left\langle\partial_{t} v+A_{v}(v), \varphi_{1}\right\rangle \\
\left(v(0), \varphi_{2}\right)_{\Omega} \\
\left\langle\partial_{t} \vartheta+A_{\vartheta}(\vartheta), \varphi_{3}\right\rangle \\
\left(\vartheta(0), \varphi_{4}\right)_{\Omega} \\
\left\langle A_{\Phi}(\Phi)+B_{\Phi}(\Phi), \varphi_{5}\right\rangle
\end{array}\right\} \forall \varphi \in Z
$$

and the operator $N: \mathcal{U}_{\text {ad }} \times \mathcal{X}_{\infty} \rightarrow \mathcal{Y}$ is given by

$$
\langle N(u, y), \varphi\rangle=\left\{\begin{array}{r}
\left\langle B_{v}(v)-F_{v}(\vartheta), \varphi_{1}\right\rangle \\
\left(-v_{0}, \varphi_{2}\right)_{\Omega} \\
\left\langle B_{\vartheta}(v, \vartheta)+G_{\vartheta}(\vartheta, \Phi)-F_{\vartheta}(u), \varphi_{3}\right\rangle \\
\left(-\vartheta_{0}, \varphi_{4}\right)_{\Omega} \\
\left\langle-F_{\Phi}(u, \vartheta), \varphi_{5}\right\rangle
\end{array}\right\} \forall \varphi \in Z,
$$


where the space $\mathcal{Y}$ is defined as in Theorem 4.3. The fact that $N(u, y) \in \mathcal{Y}$ for all $(u, y) \in \mathcal{U}_{\mathrm{ad}} \times \mathcal{X}_{\infty}$ can be seen easily from the embedding theorems for Lebesgue spaces into Morrey spaces.

Following the proof of Theorem 4.3, we find that $K \in \mathcal{L}\left(\mathcal{X}_{\infty} ; \mathcal{Y}\right)$ is invertible. Hence, we can further rewrite (4.12) equivalently as

$$
(u, y) \in \mathcal{U}_{\mathrm{ad}} \times \mathcal{X}_{\infty}: R(u, y)=y+K^{-1} N(u, y)=0 \text { in } \mathcal{X}_{\infty} .
$$

We will now show properties for the map $R: \mathcal{U}_{\mathrm{ad}} \times \mathcal{X}_{\infty} \rightarrow \mathcal{X}_{\infty}$ and conclude the theorem with the implicit function theorem as given in [50, Theorem 4.B].

Fréchet Differentiability. $\quad K^{-1}$ is continuously Fréchet differentiable due to its linearity and $N$ is continuously Fréchet differentiable due to Remark 2.1 and Lemma 2.4. Altogether we have the Fréchet differentiability of $R$, i.e., $R \in \mathcal{C}^{1}\left(\mathcal{U}_{\text {ad }} \times\right.$ $\left.\mathcal{X}_{\infty} ; \mathcal{L}\left(\mathcal{U}_{\text {ad }} \times \mathcal{X}_{\infty} ; \mathcal{X}_{\infty}\right)\right)$, where its derivative is given by

$$
\mathrm{d} R(u, y)\left[w_{u}, w_{y}\right]=w_{y}+K^{-1} \mathrm{~d} N(u, y)\left[w_{u}, w_{y}\right] .
$$

Bijectivity of $\mathrm{d}_{y} R(u, y) \in \mathcal{L}\left(\mathcal{X}_{\infty}\right)$ for all solutions $(u, y) \in \mathcal{U}_{a d} \times \mathcal{X}_{\infty}$ of (4.13). Let $(u, y) \in \mathcal{U}_{\mathrm{ad}} \times \mathcal{X}_{\infty}$ be a solution of (4.13) and $h \in \mathcal{X}_{\infty}$ be arbitrary. Then by (4.14) we have the problem

$$
r_{y} \in \mathcal{X}_{\infty}: r_{y}+K^{-1} \mathrm{~d}_{y} N(u, y)\left[r_{y}\right]=h \text { in } \mathcal{X}_{\infty}
$$

or equivalently

$$
w_{y} \in \mathcal{X}_{\infty}: K\left[w_{y}\right]+\mathrm{d}_{y} N(u, y)\left[w_{y}\right]=\mathrm{d}_{y} N(u, y)[h] \text { in } \mathcal{Y},
$$

with $w_{y}=r_{y}-h$. Arguments with embedding theorems for Lebesgue spaces into Morrey spaces easily show that $\mathrm{d}_{y} N(u, y)[h]$ belongs to $\mathcal{Y}$. Observe that $K+\mathrm{d}_{y} N(u, y)$ is precisely $L_{u, y}$ as given in (4.7). We may then apply Theorem 4.3 to deduce the existence of a solution $w_{y} \in \mathcal{X}_{\infty}$ to the above equation. Thus we have $r_{y}=w_{y}+h \in \mathcal{X}_{\infty}$ as required.

Finally, by the implicit function theorem, we obtain existence of the continuously Fréchet differentiable map $y: \mathcal{U}_{\mathrm{ad}} \rightarrow \mathcal{X}_{\infty}$ as defined in (4.11) such that the assertion regarding its derivative holds true.

4.3. Adjoint system. Employing the previous results for the linear state vectors we now study the adjoint operator and show that the adjoint problem (4.3) is indeed uniquely solvable.

Lemma 4.5. If $(v, \vartheta) \in L^{\infty}\left(I ; \mathcal{H}^{1}\right) \times L^{\infty}(Q)$ and $\left(z_{v}, z_{\vartheta}\right) \in \mathcal{V} \times V$, then

$$
\left(\nabla v^{T}-v \cdot \nabla\right) z_{v}-\vartheta \nabla z_{\vartheta} \in \mathcal{V}^{*}
$$

Proof. Let $\varphi \in \mathcal{H}^{1}$. From the embedding $\mathcal{H}^{1} \hookrightarrow L^{4}(\Omega)$, we obtain the bounds

$$
\begin{aligned}
\left|\sum_{i, j}\left\langle\left(\partial_{i} v_{j}(t)\right) z_{v, j}(t), \varphi_{i}\right\rangle\right| & \leq c_{1}\left\|\nabla v^{\mathrm{T}}(t)\right\|_{2, \Omega}\left\|z_{v}(t)\right\|_{\mathcal{H}^{1}}\|\varphi\|_{\mathcal{H}^{1}}, \\
\left|\sum_{i, j}\left\langle v_{i}(t) \partial_{i} z_{v, j}(t), \varphi_{j}\right\rangle\right| & \leq c_{2}\|v(t)\|_{\mathcal{H}^{1}}\left\|\nabla z_{v}(t)\right\|_{2, \Omega}\|\varphi\|_{\mathcal{H}^{1}}, \\
\left|\sum_{i}\left\langle\vartheta(t) \partial_{i} z_{\vartheta}, \varphi_{i}\right\rangle\right| & \leq\|\vartheta(t)\|_{\infty, \Omega}\left\|\nabla z_{\vartheta}(t)\right\|_{2, \Omega}\|\varphi\|_{\mathcal{H}^{1}},
\end{aligned}
$$


for each terms respectively, where $c_{1}, c_{2}>0$ are constants. Taking the square of

$$
\left\|\left(\nabla v^{\mathrm{T}}(t)-v(t) \cdot \nabla\right) z_{v}(t)-\vartheta(t) \nabla z_{\vartheta}(t)\right\|_{\mathcal{H}^{-1}},
$$

integrating with respect to $t$, and using the bounds from above yields

$$
\left\|\left(\nabla v^{T}-v \cdot \nabla\right) z_{v}-\vartheta \nabla z_{\vartheta}\right\|_{\mathcal{V}^{*}}^{2} \leq c\left(\|v\|_{L_{\infty}\left(I ; \mathcal{H}^{1}\right)}^{2}\left\|z_{v}\right\|_{\mathcal{V}}^{2}+\|\vartheta\|_{\infty, Q}^{2}\left\|z_{\vartheta}\right\|_{V}^{2}\right),
$$

for some constant $c>0$, thus proving the assertion.

THEOREM 4.6. Let assumptions 1-2 hold and $(u, y) \in \mathcal{U}_{a d} \times \mathcal{X}_{\infty}$. The adjoint operator $L_{u, y}^{*} \in \mathcal{L}\left(Z ; \mathcal{X}^{*}\right)$ is a homeomorphism, i.e., a unique $\tilde{z}=\left(z_{v}, z_{v, 0}, z_{\vartheta}, z_{\vartheta, 0}, z_{\Phi}\right) \in Z$ exists such that for any given $\tilde{h}=\left(h_{v}, h_{\vartheta}, h_{\Phi}\right) \in \mathcal{X}^{*}$,

$$
\tilde{z} \in Z: L_{u, y}^{*}[\tilde{z}]=\tilde{h} \quad \text { in } \quad \mathcal{X}^{*} .
$$

If further $\tilde{h} \in \mathcal{V}^{*} \times V^{*} \times\left[V^{*}\right]^{2}$, then $\tilde{z} \in Z$ obtained above can be characterized by the following problem:

$$
z \in \mathcal{X}: L_{u, y}^{*}[z]=h \quad \text { in } \quad Z^{*},
$$

where $h=\left(h_{v}, 0, h_{\vartheta}, 0, h_{\Phi}\right) \in Z^{*}$ and $z=\left(z_{v}, z_{\vartheta}, z_{\Phi}\right) \in \mathcal{X}$ with initial conditions $z_{v}(0)=$ $z_{v, 0} \in \mathcal{H}$ and $z_{\vartheta}(0)=z_{\vartheta, 0} \in L^{2}(\Omega)$, and terminal conditions $z_{v}(T)=z_{\vartheta}(T)=0$.

Proof. The first assertion follows from Theorem 4.2 together with standard arguments from functional analysis (cf. [5, Theorem 10.5]). Equation (4.16) may be explicitly written as the system

$$
z \in \mathcal{X}:\left\{\begin{aligned}
\left\langle-\partial_{t} z_{v}+A_{v}\left[z_{v}\right]+B_{v}^{*}(y)[z], \varphi_{1}\right\rangle & =\left\langle h_{v}, \varphi_{1}\right\rangle \\
\left(z_{v}(T), \varphi_{2}\right)_{\Omega} & =0 \\
\left\langle-\partial_{t} z_{\vartheta}+A_{\vartheta}\left[z_{\vartheta}\right]+B_{\vartheta}^{*}(y)[z]+G_{\vartheta}^{*}(y)[z], \varphi_{3}\right\rangle & =\left\langle h_{\vartheta}, \varphi_{3}\right\rangle \\
\left(z_{\vartheta}(T), \varphi_{4}\right)_{\Omega} & =0 \\
\left\langle A_{\Phi}^{*}\left[z_{\Phi}\right]+B_{\Phi}\left[z_{\Phi}\right]-F_{\Phi}^{*}\left[z_{\vartheta}\right], \varphi_{5}\right\rangle & =\left\langle h_{\Phi}, \varphi_{5}\right\rangle
\end{aligned}\right\} \forall \varphi \in Z
$$

where the operators $B_{v}^{*}(y), B_{\vartheta}^{*}(y), G_{\vartheta}^{*}(y), A_{\Phi}^{*}, F_{\Phi}^{*}$ for all $y \in \mathcal{X}_{\infty}$ are given by

$$
\begin{aligned}
\left\langle B_{v}^{*}(y)[z], \varphi_{1}\right\rangle & =\left\langle\left(\nabla v^{\mathrm{T}}-v \cdot \nabla\right) z_{v}-\vartheta \nabla z_{\vartheta}, \varphi_{1}\right\rangle, \\
\left\langle B_{\vartheta}^{*}(y)[z], \varphi_{3}\right\rangle & =\left\langle v \cdot \nabla z_{\vartheta}, \varphi_{3}\right\rangle, \\
\left\langle G_{\vartheta}^{*}(y)[z], \varphi_{3}\right\rangle & =\left(\sigma_{a} \mathrm{~d} I_{b}(\vartheta)\left(\mathrm{Pl}^{-1} z_{\vartheta}-z_{\Phi, 1}\right)-\operatorname{RaPr} e_{2} \cdot z_{v}, \varphi_{3}\right)_{Q}, \\
\left\langle A_{\Phi}^{*}\left[z_{\Phi}\right], \varphi_{5}\right\rangle & =\sum_{i, j}\left(\frac{\epsilon^{2}}{3 \sigma_{t}} \hat{\mathrm{a}}_{j, i} \nabla z_{\Phi, i}, \nabla \varphi_{5, j}\right)_{Q}+\left(\hat{\alpha}^{\mathrm{T}} z_{\Phi}, \varphi_{5}\right)_{\Sigma}, \\
\left\langle F_{\Phi}^{*}\left[z_{\vartheta}\right], \varphi_{5}\right\rangle & =\left(\mathrm{Pl}^{-1} \sigma_{a} z_{\vartheta} e_{1}, \varphi_{5}\right)_{Q} .
\end{aligned}
$$

Now let $\tilde{z} \in Z$ be a solution of (4.15). By formally writing the adjoint, we have

$$
\begin{aligned}
\left\langle w_{y}, L_{u, y}^{*}[\tilde{z}]\right\rangle=\left\langle w_{v},-\partial_{t} z_{v}+A_{v}\left[z_{v}\right]+B_{v}^{*}(y)[\tilde{z}]\right\rangle & +\left\langle w_{\vartheta},-\partial_{t} z_{\vartheta}+A_{\vartheta}\left[z_{\vartheta}\right]-B_{\vartheta}^{*}(y)[\tilde{z}]+G_{\vartheta}^{*}(y)[\tilde{z}]\right\rangle \\
& +\left\langle w_{\Phi}, A_{\Phi}^{*}\left[z_{\Phi}\right]+B_{\Phi}\left[z_{\Phi}\right]-F_{\Phi}^{*}\left[z_{\vartheta}\right]\right\rangle \\
=\langle & \left.\partial_{t} w_{v}+A_{v}\left[w_{v}\right]+\mathrm{d} B_{v}(y)\left[w_{y}\right]-\mathrm{d} F_{v}(y)\left[w_{y}\right], z_{v}\right\rangle+\left(w_{v}(0), z_{v, 0}\right)_{\Omega}
\end{aligned}
$$




$$
\begin{aligned}
& \quad+\left\langle\partial_{t} w_{\vartheta}+A_{\vartheta}\left[w_{\vartheta}\right]+\mathrm{d} B_{\vartheta}(y)\left[w_{y}\right]+\mathrm{d} G_{\vartheta}(y)\left[w_{y}\right], z_{\vartheta}\right\rangle+\left(w_{\vartheta}(0), z_{\vartheta}, 0\right)_{\Omega} \\
& \quad+\left\langle A_{\Phi}\left[w_{\Phi}\right]+B_{\Phi}\left[w_{\Phi}\right]-\mathrm{d}_{y} F_{\Phi}(u, y)\left[w_{y}\right], z_{\Phi}\right\rangle \\
& =\left\langle L_{u, y}\left[w_{y}\right], \tilde{z}\right\rangle
\end{aligned}
$$

which holds true for all

$$
\tilde{z} \in Z \quad \text { and } \quad w_{y} \in H^{1}\left(I ; \mathcal{H}^{1}\right) \times H^{1}\left(I ; H^{1}(\Omega)\right) \times[V]^{2}, \quad w_{y}(T)=0 .
$$

Due to Lemma 4.5 and $h \in Z^{*}$, we further have that

$$
\begin{aligned}
& \partial_{t} z_{v}:=A_{v}\left[z_{v}\right]+B_{v}^{*}(y)[\tilde{z}]-h_{v} \in \mathcal{V}^{*}, \\
& \partial_{t} z_{\vartheta}:=A_{\vartheta}\left[z_{\vartheta}\right]+B_{\vartheta}^{*}(y)[\tilde{z}]+G_{\vartheta}^{*}(y)[\tilde{z}]-h_{\vartheta} \in V^{*},
\end{aligned} \quad \text { for } \tilde{z} \in Z,
$$

which implies that $\tilde{z} \in \mathcal{W}(\mathcal{V}) \times \mathcal{H} \times \mathcal{W}(V) \times L^{2}(\Omega) \times[V]^{2}$ and thus $z \in \mathcal{X}$ with the initial and terminal conditions mentioned above, due to [43, Proposition 1.2]. The rest follows from density arguments for the mapping

$$
H^{1}\left(I ; \mathcal{H}^{1}\right) \times H^{1}\left(I ; H^{1}(\Omega)\right) \times[V]^{2} \rightarrow Z ; \quad w_{y} \mapsto\left(w_{v}, w_{v}(0), w_{\vartheta}, w_{\vartheta}(0), w_{\Phi}\right),
$$

which concludes the proof.

\section{Conclusion}

The presented analysis for the optimal boundary control of the natural convectionradiation problem yields an essential step towards the understanding of the control of applied problems involving multiphysics modeling. The increasing complexity of the models demands a careful regularity analysis to get results for the coupled problem. Further, the proof of boundedness of solutions for the $\mathrm{SP}_{3}$ model yielded a new understanding of cross-diffusion phenomena, which might be generalized for higher order $\mathrm{SP}_{N}$ models. The analytical results allow for the construction of numerical algorithms based on the adjoint information. Preliminary numerical studies can be found in [48].

\section{REFERENCES}

[1] F. Abergel and R. Temam, On some control problems in fluid mechanics, Theor. Comp. Fluid Dyn., 1, 303-325, 1990.

[2] R.A. Adams, Sobolev Spaces, New York: Academic Press, 1975.

[3] V.I. Agoshkov and C. Bardos, Optimal control approach in inverse radiative transfer problems: The problem on boundary function, ESAIM, Control Optim. Calc. Var., 5, 259-278, 2000.

[4] V.I. Agoshkov, E.I. Parmuzin, V.P. Shutyaev, and C. Bardos, Numerical analysis of iterative algorithms for an inverse boundary transport problem, Math. Mod. Meth. Appl. Sci., 10(1), $11-29,2000$.

[5] H.W. Alt, Lineare Funktionalanalysis, 5. Auflage, Springer-Verlag Berlin Heidelberg, 2006.

[6] H. Amann, Maximum principles and principal eigenvalues, Ten Mathematical Essays on Approximation in Analysis and Topology, Elsevier B.V., 2004.

[7] G. Bärwolff and M. Hinze, Optimization of semiconductor melts, Z. Angew. Math. Mech., 86, 423-437, 2006.

[8] A. Belmiloudi, Robin-type boundary control problems for the nonlinear Boussinesq type equations, J. Math. Anal. Appl., 273, 428-456, 2002.

[9] J.L. Bolderini, E. Fernandéz-Cara, and M.A. Rojas-Medar, An optimal control problem for a generalized Boussinesq model: The time dependent case, Rev. Mat. Comput., 20, 339-366, 2007.

[10] J. Boussinesq, Theorie Analytique de la Chaleur, Gauthier-Villars, Paris, 2, 1903.

[11] S. Carl, V.K. Le, and D. Motreanu, Nonsmooth variational problems and their inequalities, Springer Science + Business Media B.V., 2010.

[12] M.K. Choudhary and N.T. Huff, Mathematical modelling in the glass industry: An overview of status and needs, Glastech. Ber. Glass Sci. Technol., 70, 363-370, 1997. 
[13] D. Clever and J. Lang, Optimal control of radiative heat transfer in glass cooling with restrictions on the temperature gradient, Optim. Contr. Appl. Met., 33, 157-175, 2011.

[14] A. Draoui, F. Allard, and C. Beghein, Numerical analysis of heat transfer by natural convection and radiation in participating fluids enclosed in square cavities, Num. Heat Transfer, Part A, 20, 253-261, 1991.

[15] D. Gilbarg and N.S. Trudinger, Elliptic Partial Differential Equations of Second Order, Springer-Verlag, Berlin, First Edition, 1983.

[16] J.A. Griepentrog, Linear elliptic boundary value problems with non-smooth data: Campanato spaces of functionals, Math. Nachr., 243, 19-42, 2002.

[17] J.A. Griepentrog, Maximal regularity for non-smooth parabolic problems in Sobolev-Morrey spaces, Adv. Diff. Equ., 12, 1031-1078, 2007.

[18] J.A. Griepentrog, Sobolev-Morrey spaces associated with evolution equations, Adv. Diff. Equ., $12,781-840,2007$.

[19] J.A. Griepentrog and L. Recke, Linear elliptic boundary value problems with non-smooth data: Normal solvability on Sobolev-Campanato spaces, Math. Nachr., 225, 39-74, 2001.

[20] R. Griesse, M. Hintermüller, and M. Hinze, Differential stability of control constrained optimal control problems for the Navier-Stokes equations, Num. Func. Anal. Opt., 26, 829-850, 2005.

[21] M. Hintermüller and M. Hinze, A SQP-semismooth Newton-type algorithm applied to control of the instationary Navier-Stokes system subject to control constraints, SIAM J. Control Optim., 16, 1177-1200, 2006.

[22] M. Hinze, Optimal and Instantaneous Control of the Instationary Navier-Stokes Equations, Habilitation Thesis, Technische Universität Berlin, Berlin, Germany, 2000.

[23] M. Hinze and K. Kunisch, Second order methods for boundary control of the instationary Navier-Stokes system, Z. Angew. Math. Mech., 84, 171-187, 2004.

[24] M. Hinze and K. Kunisch, Second order methods for optimal control of time-dependent fluid flow, SIAM J. Control Optim., 40, 925-946, 2001.

[25] M. Hinze, R. Pinnau, M. Ulbrich, and S. Ulbrich, Optimization with PDE Constraints, Springer Science + Business Media B.V., 2009.

[26] A. Kufner, O. John, and S. Fuvcík, Function Spaces, Publishing House of the Czechoslovak, Academy of Sciences, Prague, 1977.

[27] O.A. Ladyvzenskaya, On new estimates for the Navier-Stokes equations and globally stable attractors, J. Math. Sci., 77(3), 3199-3206, 1995.

[28] O.A. Ladyvzenskaya, The Mathematical Theory of Viscous Incompressible Flow, Gordon and Breach, New York, Second Edition, 1969.

[29] O.A. Ladyvzenskaya, V.A. Solonnikov, and N.N. Ural'ceva, Linear and Quasi-linear Equations of Parabolic Type, American Mathematical Society, Translations of Mathematical Monographs, 23, 1968.

[30] E.W. Larsen, J.E. Morel, and J. McGhee, Asymptotic Derivation of the Simplified $P_{n}$ Equations, Proceedings of Joint International Conference on Mathematical Methods and Supercomputing in Nuclear Applications, Karlsruhe, Germany, 1993.

[31] E.W. Larsen, G. Thömmes, A. Klar, M. Seaïd, and T. Götz, Simplified $P_{N}$ approximations to the equations of radiative heat transfer and applications, J. Comput. Phys., 183, 652-675, 2002.

[32] C. Meyer, Second-order sufficient optimality conditions for a semilinear optimal control problem with nonlocal radiation interface conditions, ESAIM Control Optim. Calc. Var., 13(4), 750-775, 2007.

[33] C. Meyer, P. Philip, and F. Tröltzsch, Optimal control of a semilinear PDE with nonlocal radiation interface conditions, SIAM J. Control Optim., 45, 688-721, 2006.

[34] C. Meyer and I. Yousept, Regularization of state-constrained elliptic optimal control problems with nonlocal radiation interface conditions, Comput. Optim. Appl., 44(2), 183-212, 2009.

[35] J.M. Milhaljan, A rigorous exposition of the Boussinesq approximations applicable to a thin layer of fluid, Astrophysics J., 136, 1126, 1962.

[36] M.F. Modest, Radiative Heat Transfer, McGraw-Hill Series in Mechanical Engineering, McGraw-Hill, Inc., 1993.

[37] A. Oberbeck, Über die Wärmerleitung der Flussigkeiten bei der Berücksichtigung der Strömungen infolge von Temperaturdifferenzen, Ann. Phys. Chem., 7, 271, 1879.

[38] R. Pinnau, Analysis of optimal boundary control for radiative heat transfer modeled by the SP1-system, Commun. Math. Sci., 5(4), 951-969, 2007.

[39] R. Pinnau and A. Schulze, Newton's method for optimal temperature-tracking of glass cooling processes, Inverse Probl. Sci. Eng., 15(4), 303-323, 2007.

[40] R. Pinnau and M. Seaid, Simplified $P_{N}$ models and natural convection-radiation, Progress in 
Industrial Mathematics at ECMI 2006, 397-401, Math. Ind., 12, Springer, Berlin, 2008.

[41] R. Pinnau and G. Thömmes, Optimal boundary control of glass cooling processes, Math. Meth. Appl. Sci., 27(11), 1261-1281, 2004.

[42] M. Renardy and R.C. Rogers, An Introduction to Partial Differential Equations, SpringerVerlag New York, Inc., 2004.

[43] R.E. Showalter, Monotone Operators in Banach Space and Nonlinear Partial Differential Equations, Mathematical Surveys and Monographs, 49, 1997.

[44] R. Temam, Navier-Stokes Equations, Studies in Mathematics and its Applications, NorthHolland Publishing Company, 2, 1977.

[45] R. Temam, Infinite-dimensional dynamical systems in mechanics and physics, Springer-Verlag, New York, Second Edition, 1997.

[46] G. Thömmes, R. Pinnau, M. Seaid, T. Götz, and A. Klar, Numerical methods and optimal control for glass cooling processes, Transp. Theory Stat. Phys., 31(4-6), 513-529, 2002.

[47] F. Tröltzsch, Optimal Control of Partial Differential Equations: Theory, Methods, and Applications, Amer. Math. Soc., 2010.

[48] O. Tse, $S P_{N}$ systems in Radiative Heat Transfer and Natural Convection-Radiation Models, PhD Thesis, TU Kaiserslautern, 2011.

[49] O. Tse, R. Pinnau, and N. Siedow, Identification of temperature dependent parameters in laser-interstitial thermo therapy, Math. Mod. Meth. Appl. Sci., 22(9), 1250019, 2012.

[50] E. Zeidler, Nonlinear Functional Analysis and Its Applications I: Fixed-Point Theorems, Springer-Verlag New York, Inc., 1986.

[51] E. Zeidler, Nonlinear Functional Analysis and Its Applications II/A: Linear Monotone Operators, Springer-Verlag, New York, 1990. 